\title{
ANÁLISIS MICROMORFOLÓGICOS PARA LA RECONSTRUCCIÓN DE ACTIVIDADES EN EL COMPLEJO ARQUITECTÓNICO DE MARANGA-LIMA
}

\author{
MICROMORPHOLOGICAL ANALYSES FOR THE RECONSTRUCTION OF \\ ACTIVITIES IN THE MARANGA-LIMA ARCHITECTURAL COMPLEX
}

\author{
Rafael Vega Centeno ${ }^{1}$ y Carol Lang ${ }^{2}$
}

\begin{abstract}
Se presentan los primeros resultados de análisis micromorfológicos llevados a cabo en cuatro muestras extraídas de pisos de ocupación del complejo urbano de Maranga-Lima (ca. 350-780 DC). Los resultados de dichos análisis revelan evidencias de áreas de actividad que involucran eventos de combustión donde, junto con material carbonizado, aparecen componentes orgánicos (en particular restos óseos y malacológicos). Asimismo, se ha recuperado la evidencia de una posible cobertura del piso con esteras, inferidas por la concentración de fitolitos de pastos no carbonizados. Así, el estudio pone en evidencia el potencial de los análisis micromorfológicos para la reconstrucción de áreas de actividad en los espacios del complejo urbano que suelen encontrarse "limpios" en perspectiva macroscópica.
\end{abstract}

Palabras claves: micromorfología, microestratigrafía, sedimentación, procesos edafológicos, Cultura Lima, arquitectura.

We present the first results of micromorphological analyses conducted on four samples extracted from occupational floors of the Maranga-Lima urban complex (AD 350-780). Results reveal evidence of activity areas that involved firing events, with the presence of carbonized material and organic elements (particularly bone and shell). In addition, we recovered evidence of possible floor matting due to the concentration of non-carbonized grass phytoliths. Thus, this study notes the potential of micromorphological analyses for the reconstruction of activity areas in urban spaces that are usually found to be "clean" from a macroscopic perspective.

Key words: Micromorphology, microstratigraphy, sedimentation, pedogenesis, Lima Culture, architecture.

Es un axioma en arqueología que la reconstrucción de áreas de actividad descansa fundamentalmente en el hallazgo de restos de dichas actividades en determinados espacios o superficies. Por otro lado, es también cierto que el hallazgo de dichos restos resulta con frecuencia problemático debido a su escasez en los lugares de su uso original. Más aún, las áreas donde se llevaron a cabo las actividades suelen encontrarse "limpias" de basura, haciendo más difícil nuestra reconstrucción de ellas (La Motta y Schiffer 1999; Schiffer 1995:31-33). Esta situación es incluso más recurrente en el caso de espacios arquitectónicos vinculados con actividades rituales o administrativas.

El caso de los edificios asignados a la Cultura Lima (ca. 200-750 DC) es un ejemplo típico de esta situación. Recientes investigaciones han permitido ampliar significativamente nuestro conocimiento de los espacios arquitectónicos vinculados a los edificios públicos de esta cultura y, en ciertas ocasiones, el hallazgo singular de restos sobre pisos y rellenos ha permitido identificar actividades puntuales de consumo conspicuo vinculadas con eventos constructivos. Sin embargo, aún es difícil precisar el tipo de actividades regulares o cotidianas en dichos espacios.

Frente a este escenario, decidimos incorporar, dentro de nuestras investigaciones en el Complejo Maranga, un estudio exploratorio de microestratigrafía y micromorfología a partir de cuatro muestras de pisos ocupacionales asociados con el complejo urbano allí existente durante la época Lima. Se trata de análisis que tienen el potencial de ampliar significativamente el registro de restos artefactuales y ecofactuales vinculados con superficies de ocupación. Por lo tanto, ofrecen el potencial para expandir nuestras posibilidades de interpretación de las actividades llevadas a cabo en los espacios arquitectónicos Lima.

1 Departamento de Humanidades, Pontificia Universidad Católica del Perú, Lima, Perú. rvegacen@ gmail.com

2 Department of Archaeology, University of York, UK. langgeoarchaeology@gmail.com 
Es así que presentamos en las siguientes líneas los resultados de dichos análisis y las perspectivas que nos ofrecen para futuras investigaciones.

\section{Los Complejos Arquitectónicos de la Cultura Lima}

Desde finales del siglo XIX y a lo largo del siglo $\mathrm{XX}$, diversos reconocimientos e investigaciones permitieron definir que, durante el periodo Intermedio Temprano (ca. 200 AC-550 DC), la costa central del Perú experimentó la construcción de edificios públicos de gran escala vinculados a la que fue conociéndose como Cultura Lima (Flores 2005; Franco y Paredes 2001; Hutchinson 1873:276-280; Jijón y Caamaño 1949; Kroeber 1956; Lumbreras 2011; Middendorf 1973 [1894]; Stumer 1954; Uhle 1998 [1910]). Se sabe mucho menos, sin embargo, de los espacios que circundaban dichos edificios, debido en parte tanto a problemas de conservación como a la cobertura de dichos espacios, primero por el avance agrícola y, posteriormente, por el avance urbano.

Existen, sin embargo, algunos sitios en los que se ha podido documentar el espacio construido alrededor de los edificios públicos. Uno de estos sitios es Cerro Culebras, en el Valle del Chillón. Allí se registró una pirámide trunca de unos 65 x $40 \mathrm{~m}$ de área que se encontraba circunscrita a un conjunto de patios y cercaduras de unos $250 \times 150 \mathrm{~m}$. Este conjunto se encontraba delimitado por un muro perimetral y, a su interior, se podían diferenciar nueve espacios de distintos tamaños y formas que rodean a la pirámide (Engel 1987:Fig.18d; Paredes 1992) (Figura 1a). Si bien no se han registrado los sistemas de acceso o las formas de desplazamiento al interior de dichos espacios, las evidencias superficiales ponen de manifiesto la importancia de este conjunto arquitectónico para el funcionamiento y existencia de la estructura piramidal, en tanto que regulaban el acceso a esta y debieron ser espacios de actividades directamente relacionadas con la misma.

Se ha registrado un diseño arquitectónico muy semejante al de Cerro Culebras en el Conjunto Tello del sitio de Cajamarquilla. El Conjunto Tello cubre un área de 450 x 400 m, y está delimitado también por un muro perimetral. En la parte central del conjunto se encuentra una pirámide de unos 100 x $90 \mathrm{~m}$. Como en el caso de Cerro Culebras, el conjunto alberga un grupo de recintos y patios organizados en disposición ortogonal alrededor de la pirámide (Segura 2001:32) (Figura 1b).
En los dos casos citados, fue posible registrar el complejo arquitectónico en su integridad. En otros sitios, si bien no ha sido posible registrar todo el conjunto, se ha podido definir la existencia de conjuntos arquitectónicos adyacentes a los edificios piramidales, como en el caso de Huaca Pucllana. Allí existe una estructura de unos $250 \times 100$ m rodeada por las calles y viviendas de la ciudad de Lima, salvo en su lado noroeste, donde es posible registrar un área de unos 175 x $150 \mathrm{~m}$ de patios, plazas y recintos (Flores 2005:33) (Figura 1c). Si el conjunto que rodeaba a Huaca Pucllana fue proporcionalmente similar al de Cerro Culebras o Cajamarquilla, podría haber cubierto un área de hasta 37 ha.

Evidencias de muros formales sugieren la existencia de conjuntos similares alrededor de estructuras piramidales en los sitios de Cerro Trinidad (Willey 1943:Fig. 02), Maranga (Narváez y Manrique 2014:101-106) y Catalina Huanca (Maquera y Esteban 2014). En ese sentido, parece ser que dentro de los patrones arquitectónicos Lima, las estructuras piramidales se ubicaban siempre dentro de un conjunto arquitectónico más extenso, que delimitaba espacios para actividades probablemente vinculadas con estos y, por otro lado, regulaba su accesibilidad. De ser así, no sería posible entender la naturaleza y funcionamiento de dichos edificios sin abordar la naturaleza de los espacios arquitectónicos que los circundaban.

Son pocos, lamentablemente, los espacios de este tipo que han sido estudiados en detalle. En el caso de Cerro Culebras, estos espacios no han sido excavados aún. En el caso del Conjunto Tello de Cajamarquilla, se llevaron a cabo excavaciones en el patio adyacente al frontis de la pirámide. Estas excavaciones permitieron definir una secuencia de al menos cuatro pisos, donde lo más relevante fue el hallazgo de pozos y depresiones interpretados como desechos de actividades de producción y consumo de chicha (Segura 2001:35-45). No se cuenta, sin embargo, con una delimitación precisa del espacio en el que se llevaron a cabo estas actividades o de sus posibles cambios a lo largo del tiempo.

Huaca Pucllana es, sin duda, el lugar donde este tipo de espacios se han estudiado con más detalle. La zona de arquitectura adyacente a la pirámide ha sido denominada Sector Noreste. Allí se definió un tipo de espacio característico como es el "patio con estructuras escalonadas"; áreas delimitadas por muros en cuyo interior pueden encontrarse una o dos banquetas y que con frecuencia incluyen numerosos huecos de poste que sugieren una palizada y un techado (Flores 


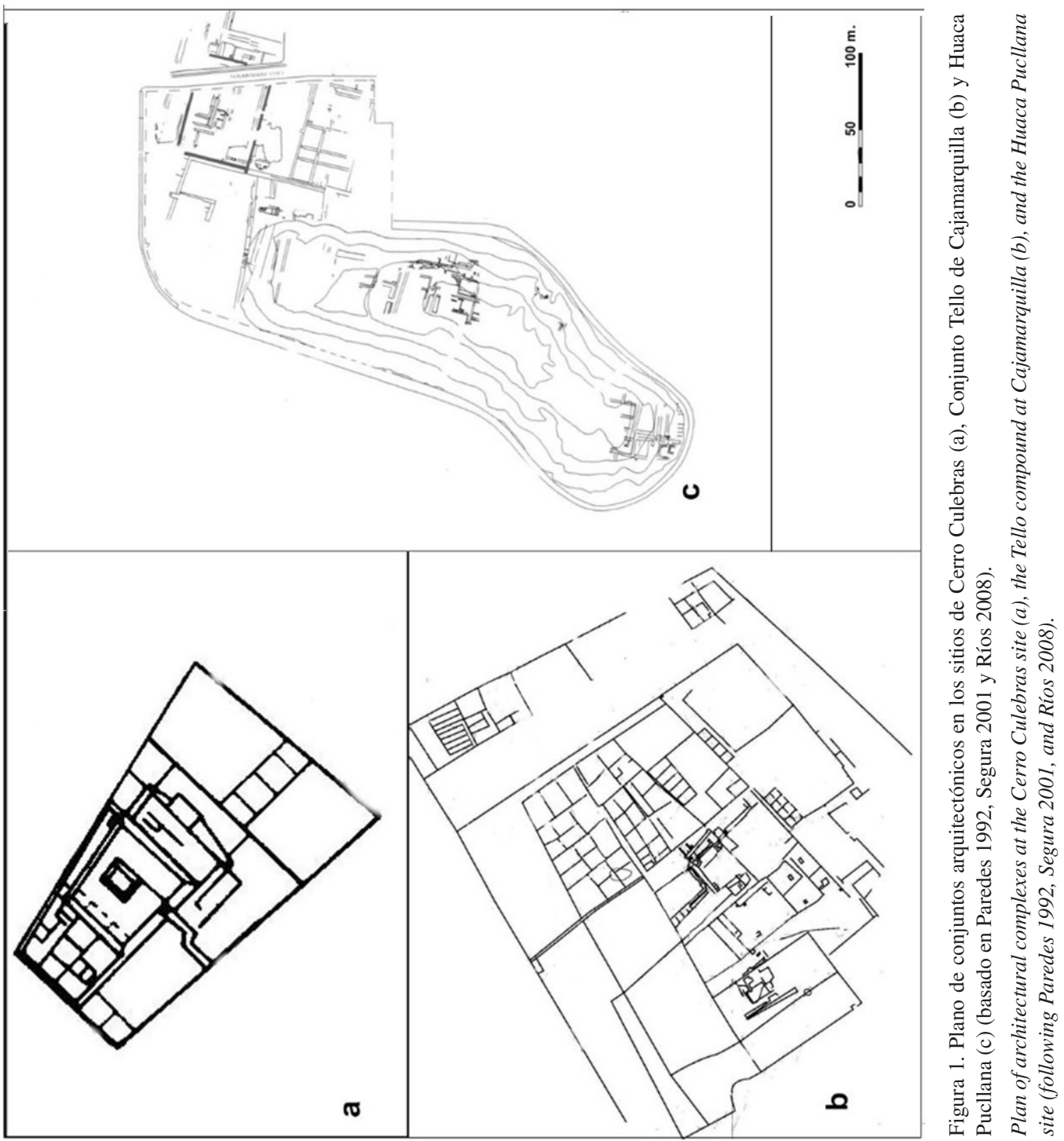


et al. 2012). Se ha reportado, a su vez, que el número, tamaño y organización de estos patios cambió a lo largo de tres fases dentro de este conjunto.

Las evidencias de Pucllana han permitido, asimismo proponer la existencia de sistemas de organización de los espacios, que incluyen, por ejemplo, sistemas de recintos y patios articulados con pasadizos, sistemas de patios con banquetas y sistemas de accesos con rampas, recintos, pasadizos, patios y plazas interconectados (Vargas 2012:305). Fue notable también el hallazgo, en una de las plazas con banquetas, de una acumulación de basura que incluye restos orgánicos, cerámica, artefactos líticos, entre otros. Dicha acumulación se encontró depositada inmediatamente antes del inicio de una etapa constructiva y ha sido interpretada como los restos de un gran evento de consumo de alimentos previos a la actividad de construcción (Ríos 2008).

Este caso, al igual que el del Conjunto Tello, nos presenta evidencias de actividades vinculadas con eventos constructivos y ha permitido evaluar la importancia de la convocatoria a festines como mecanismo de reclutamiento de mano de obra dentro de las estrategias políticas de las élites Lima. No existen, sin embargo, evidencias comparables de las actividades cotidianas que se habrían efectuado en dichos espacios. Esto se debe, en gran medida, a que, como en el caso de Huaca Pucllana, los contextos excavados revelaron pisos y espacios fundamentalmente limpios y, por lo tanto, se carece de información artefactual o ecofactual que permita reconstruir las áreas de actividad al interior de los patios, recintos y plazas que rodeaban a los edificios públicos.

En ese sentido, se hace necesario incorporar, dentro de la investigación sobre los espacios públicos Lima, nuevas aproximaciones y técnicas analíticas que permitan ir más allá de los registros arqueológicos convencionales. Resulta de particular interés el uso de técnicas que incrementen la resolución de nuestro registro, yendo más allá de las evidencias macroscópicas. Es en esa línea que se considera el potencial de los análisis microestratigráficos y micromorfológicos para el estudio de las superficies de ocupación en espacios arquitectónicos Lima.

\section{Análisis Microestratigráficos y Micromorfológicos en Arqueología}

La microestratigrafía es la observación de suelos y sedimentos en muestras no alteradas de tierra, recuperadas del campo y convertidas en secciones delgadas susceptibles de ser observadas en microscopios de diferentes magnitudes. Se trata de una técnica derivada de la petrografía y que, a partir de la década de 1930, tuvo un uso amplio como herramienta de análisis edafológico (Goldberg 1983:139). Fue en el último cuarto del siglo XX que el registro microestratigráfico, como recurso del estudio micromorfológico, comenzó a ser usado en la investigación arqueológica. Las primeras aproximaciones se orientaban a comparar sedimentaciones naturales y culturales, con el objetivo de identificar ocupaciones humanas y establecer su ubicación cronológica dentro de contextos geomorfológicos regionales (Goldberg 1983:149). Con el tiempo, fueron explorándose nuevas potencialidades de esta técnica, como la posibilidad de reconstruir formaciones microscópicas de sedimentos y suelos o procesos tafonómicos que contribuían a expandir el entendimiento de la historia ocupacional de los sitios (Amadio 2019; Karkanas y Goldberg 2007; Macphail et al. 1989). En este proceso, la técnica adquirió mayor relevancia cuando se reconoció su capacidad para contribuir en la identificación de componentes arquitectónicos como techos (Friesem et al. 2013) o la identificación de determinadas actividades humanas (Courty 1992).

Las potencialidades del análisis micromorfológico en el estudio de contextos arquitectónicos fue puestasen evidencia en las investigaciones del sitio de Çatal Höyuk, un famoso asentamiento neolítico de la península de Anatolia (Boivin 2000; Matthews et al. 1997; Shillito et al. 2011). En dichos trabajos se confirmó que, junto con la reconstrucción de procesos edafológicos de origen antrópico, era posible reconstruir o incrementar nuestra capacidad de reconstruir áreas de actividad (Matthews et al. 1997:283). El análisis micromorfológico incluyó el examen de la deposición de sedimentos; su estructura y organización de partículas; grados de compactación y porosidad; grado de homogeneidad o heterogeneidad, entre otras variables. Incluyó además la identificación de componentes minerales, sus dimensiones y forma, así como de componentes bioarqueológicos, desde huesos, valvas de moluscos, coprolitos y; diferentes materiales botánicos, en forma de carbón, ceniza, fitolitos, etc. Incluyó, por último, la identificación de restos de artefactos de piedra, cerámica, adobe, metal, etc. (Matthews et al. 1997:287-288).

Un aspecto importante de la aplicación de esta técnica en Çatal Höyuk fue que su recojo proviniese de unidades arquitectónicas definidas, respaldadas además por un buen registro de secuencias deposicionales. Era 
importante, en tal sentido, una visión preliminar del contexto de extracción (zonas techadas o al aire libre, instalaciones de cocina, almacenamiento, descanso o de uso ritual) y una eficiente estrategia de muestreo (Matthews et al. 1997:284, 291).

Fue así que el estudio de microestratigrafía y micromorfología en el sitio de Çatal Höyuk logró expandir notablemente la información sobre procesos de formación de depósitos y basurales, identificando la frecuencia del barrido y trapeado de pisos, la remoción y apisonamiento de micro-desperdicios (Shillito et al. 2011), el enlucido cíclico de paredes y pisos (Boivin 2000) e incluso el alfombrado de estos últimos (Matthews 2005). Puede decirse que nuestra visión sobre la vida cotidiana y el uso de los espacios en Çatal Höyuk ha cambiado radicalmente luego de las aproximaciones microestratigráficas.

Los estudios de micromorfología vienen siendo aplicados con éxito también en el ámbito sudamericano. Su uso para el análisis de conchales en Tierra del Fuego permitió la identificación de huellas de apisonados e incluso de preparación de piso para viviendas de material perecible (Balbo et al. 2010). La micromorfología ha permitido también una expansión del conocimiento del contexto geoarqueológico de las primeras ocupaciones humanas en la Patagonia occidental (Méndez et al. 2018). En el sur de Sudamérica se ha avanzado, además, en la construcción de una colección de referencia de microsedimentos provenientes de playas, bosques y emplazamientos cercanos a sitios arqueológicos, que ampliará la comprensión de muestras provenientes de dichos sitios (Villagran et al. 2011).

Trabajos similares se han realizado en los conchales de las costas del Brasil, llevando a replantear la interpretación de este tipo de sitios, previamente considerados como depósitos secundarios de basura con ocupaciones marginales. Las evidencias microestratigráficas han permitido identificar, dentro de los conchales, asentamientos con superficies apisonadas, espacios domésticos delimitados e, incluso, eventos de abandono (Villagran 2019). La micromorfología ha permitido, además, abordar desde los conchales, el estudio de cambios en el medio ambiente costero a partir de micro-bioindicadores (Villagran y Gianini 2014).

Estudios semejantes vienen permitiendo el registro del desarrollo de la agricultura en la Amazonía, a partir del registro de evidencias de tala y quema, así como de las secuencias ocupacionales en montículos de tierra ubicados en Uruguay (Villagrán 2016). El estudio de suelos agrícolas a través de la micromorfología ha sido aplicado incluso en un contexto andino como el Valle de Paca, permitiendo distinguir las propiedades de los suelos de terrazas agrícolas y sus procesos de degradación (Goodman-Elgar 2008).

La micromorfología es, en ese sentido, una aproximación en proceso de expansión tanto en término de temas de estudio como en sus posibilidades de aplicación. Por su parte, de acuerdo con lo planteado anteriormente, puede resultar fundamental para expandir nuestra capacidad de registro en contextos arquitectónicos que suelen presentarse limpios a nivel macroscópico. Resulta, por lo tanto, promisorio evaluar su potencial en contextos andinos y, en particular, dentro de contextos arquitectónicos Lima de la costa central. En ese sentido, conducimos el análisis de cuatro muestras provenientes del sitio complejo arquitectónico Lima de Maranga para evaluar su potencial. Este caso es el que se presenta a continuación.

\section{El Complejo Maranga-Lima}

El Complejo Maranga-Lima se encuentra ubicado en la margen sur del valle bajo del Rímac, entre el distrito de San Miguel y el Cercado de Lima (Figura 2). Se trata de un sitio de más de 150 has en cuyo interior destacan cuatro montículos plataforma de gran escala y 10 montículos de menor tamaño (Figura 3). El sitio se ubica en la actualidad dentro de Lima Metropolitana, entre terrenos del zoológico municipal, dos universidades y urbanizaciones. Se trata sin duda del asentamiento más extenso de la Cultura Lima.

Si bien la mayor parte de Maranga se encuentra debajo de las construcciones de la Lima moderna, existen importantes extensiones de terreno libres donde es posible estudiar las características de la organización espacial del complejo urbano que rodeaba a las estructuras piramidales. Una de esas extensiones se encuentra en los terrenos del Zoológico - Parque de las Leyendas (PATPAL), entre las Huacas 31 (Huaca Larga) y 20a (Huaca El Rosal). Se trata de alrededor de 1,5 ha limitadas al oeste por Huaca 31, al norte por el muro perimetral del PATPAL (hacia la Urbanización Pando Etapa 9), al este por Huaca 20a y el muro perimetral del PATPAL (hacia la Avenida Riva Agüero) $y$, al sur, por las instalaciones del PATPAL (Figura 4). En esta área, la excavación de una zanja en la segunda mitad del Siglo XX cerca de Huaca 31 puso en evidencia la existencia de muros y pisos 


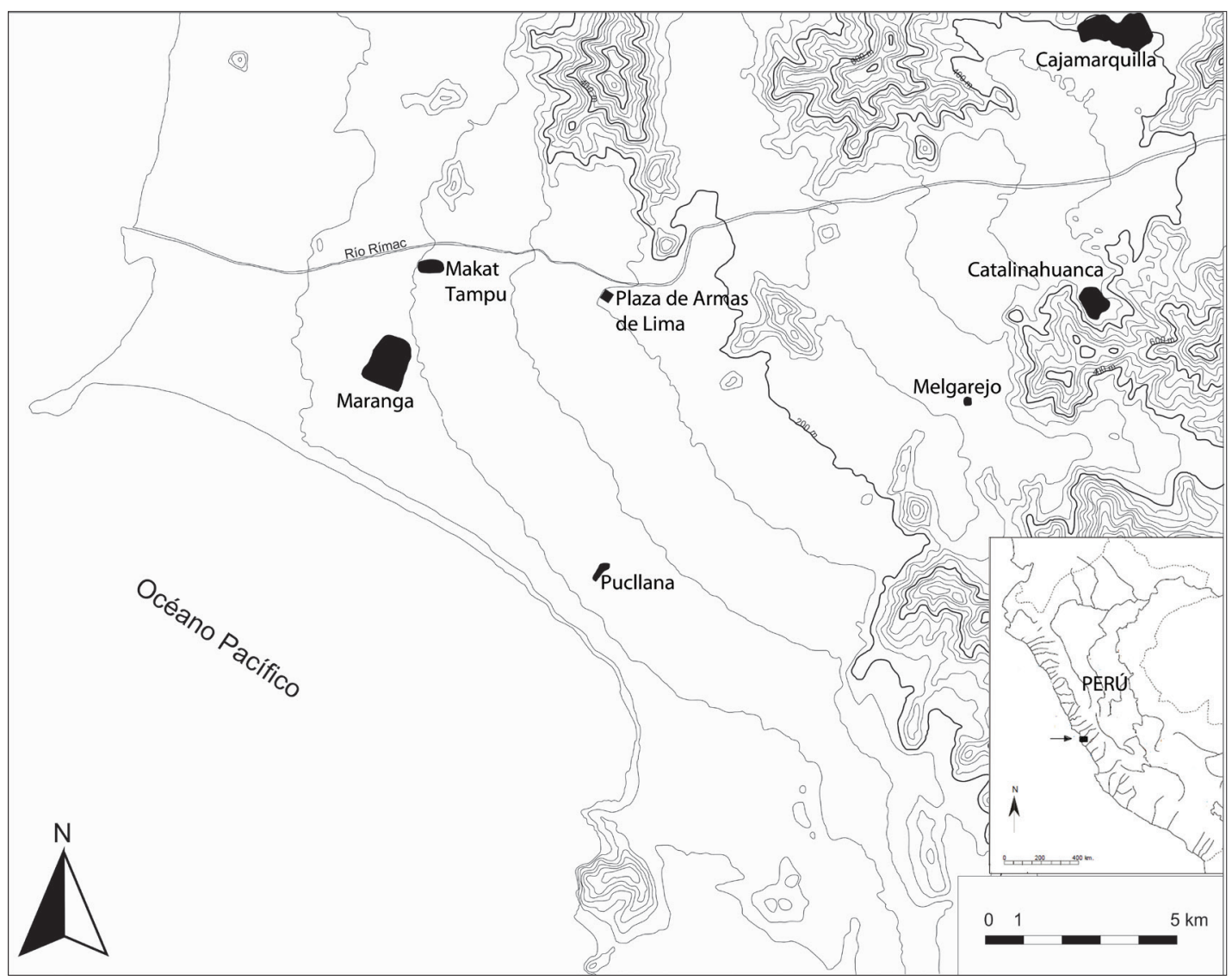

Figura 2. Plano del valle bajo del Rímac.

Plan of the Rimac Lower Valley.

entre 1,5 y $2 \mathrm{~m}$ bajo la superficie; componentes que aparecieron en pequeñas excavaciones adyacentes (Narváez y Manrique 2014:101-106). Posteriores trabajos de evaluación confirmaron la existencia de componentes arquitectónicos a lo largo y ancho de los terrenos ubicados entre ambas huacas (Carrión 2006). Nuestros trabajos en el área de la zanja adyacente a la Huaca 31 permitieron identificar una secuencia de seis pisos en asociación con un muro y banqueta, seguidos por eventos de colapso y reocupación del área (Vega Centeno et al. 2021).

Estos antecedentes ponían en evidencia la existencia de espacios arquitectónicos en el área entre los dos montículos en cuestión, constituyéndose en un espacio único para entender la organización espacial de Maranga. En esa línea, se diseñó una excavación exploratoria para evaluar las características de dichos espacios y las posibilidades de estudio mediante técnicas analíticas como la micromorfología. La zona de excavación se ubicó en la explanada adyacente al lado este de Huaca 31, a unos $60 \mathrm{~m}$ al norte de la zanja antes descrita (Figura 4). Se trató de un área de 10 x $8 \mathrm{~m}$, dividida en cuatro unidades de excavación de $20 \mathrm{~m}^{2}$. De acuerdo con la disponibilidad de tiempo y recursos, se excavaron dos de ellas (Unidades 1 y 4).

La estratigrafía en estas unidades (Figura 5) incluye sedimentaciones contemporáneas (Capas A y B) de unos $27 \mathrm{~cm}$ de espesor. Bajo estos sedimentos se encuentra un suelo agrícola que descansa en una capa limosa de unos $43 \mathrm{~cm}$ de espesor en promedio (Capa C). Es bajo esta capa que aparece la primera superficie de ocupación precolombina, caracterizada por extensiones de basura y ceniza junto con hoyos de basura. Esta superficie se encuentra sobre una capa limo-arenosa de espesor muy variable (Capa E), compuesta al parecer por adobes y mortero de muros colapsados y desintegrados. Bajo esta capa apareció otra superficie de ocupación, que incluía alineamientos de 


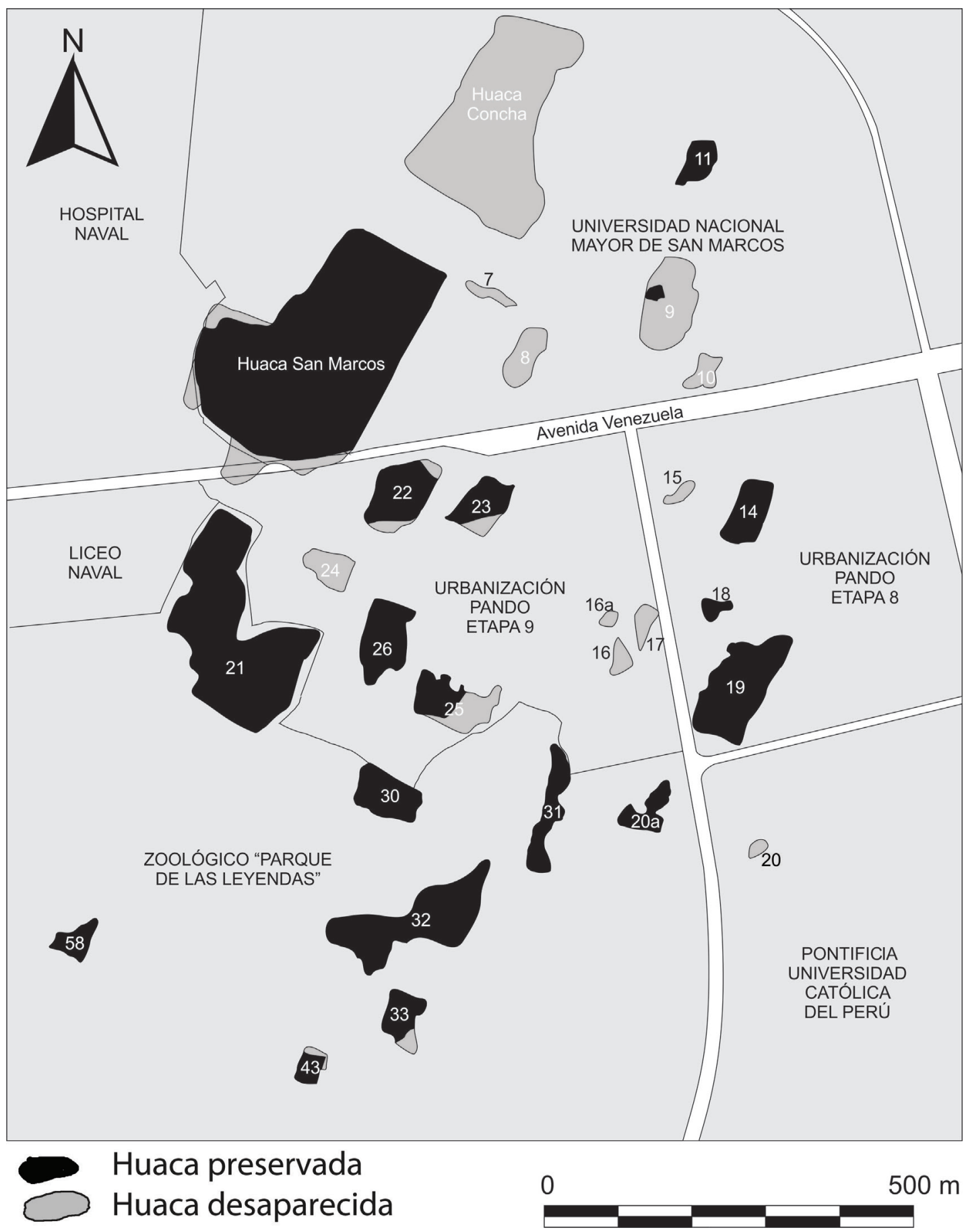

Figura 3. Plano del Complejo Arqueológico de Maranga en época de la Cultura Lima. Plan of the Maranga Archaeological Complex during the Lima Culture. 


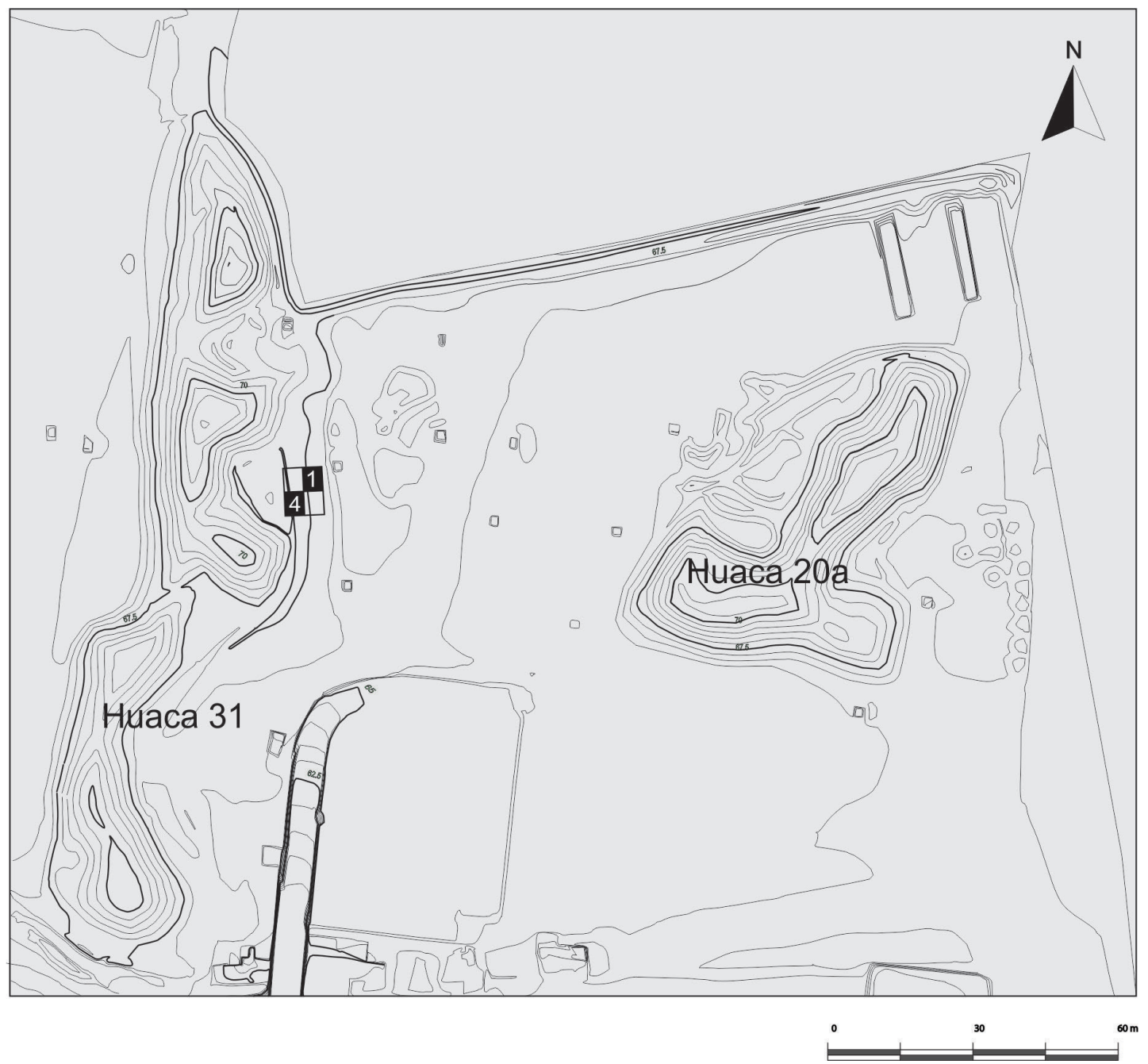

Figura 4. Plano de la zona de Maranga entre Huaca 31 (Huaca Larga) y Huaca 20a (Huaca El Rosal) con ubicación de las unidades excavadas.

Plan of the Maranga sector between Huaca 31 (Huaca Laga) and Huaca 20a (Huaca El Rosal), including the location of excavations.

adobes reutilizados como parte de pequeñas estructuras habitacionales. Esta superficie descansaba sobre una capa limo-arenosa con presencia de cantos rodados (algunos de 30 a $35 \mathrm{~cm}$ de largo), trozos de adobes y abundante material cultural (Capa F) formada al parecer por desplazamientos aluviales de mediana intensidad. Al parecer, las superficies registradas sobre las capas $\mathrm{F}$ y E corresponden a reocupaciones del área luego del colapso de las estructuras del complejo en el marco de eventos aluviales intensos.

Bajo la capa $\mathrm{E}$, se pudo registrar el último piso de la ocupación formal del Complejo Maranga en este sector (Capa G). En la Unidad 1 (Figuras 6 y 7), este piso incluía una banqueta hacia el norte, una plataforma de adobes hacia el perfil este y una rampa ascendente en el lado sur. Significativamente, en la Unidad 4, este piso se encontraba a unos $20 \mathrm{~cm}$ por encima del nivel de la Unidad 1, hecho consistente con la presencia de la rampa. Por otro lado, al interior de la Unidad 4 (Figura 8), fue posible identificar otro muro de contención, desplazado en dirección E-W, de unos $35 \mathrm{~cm}$ de altura, que generaba la existencia de otra banqueta, ubicada en el lado sur de la Unidad. El relleno de esta banqueta (Capa Ñ) fue parcialmente excavado, revelando una gran cantidad de adobes reutilizados como parte del mismo.

Si bien se trató de excavaciones de extensión limitada, la existencia de pisos, rampas pequeñas y 


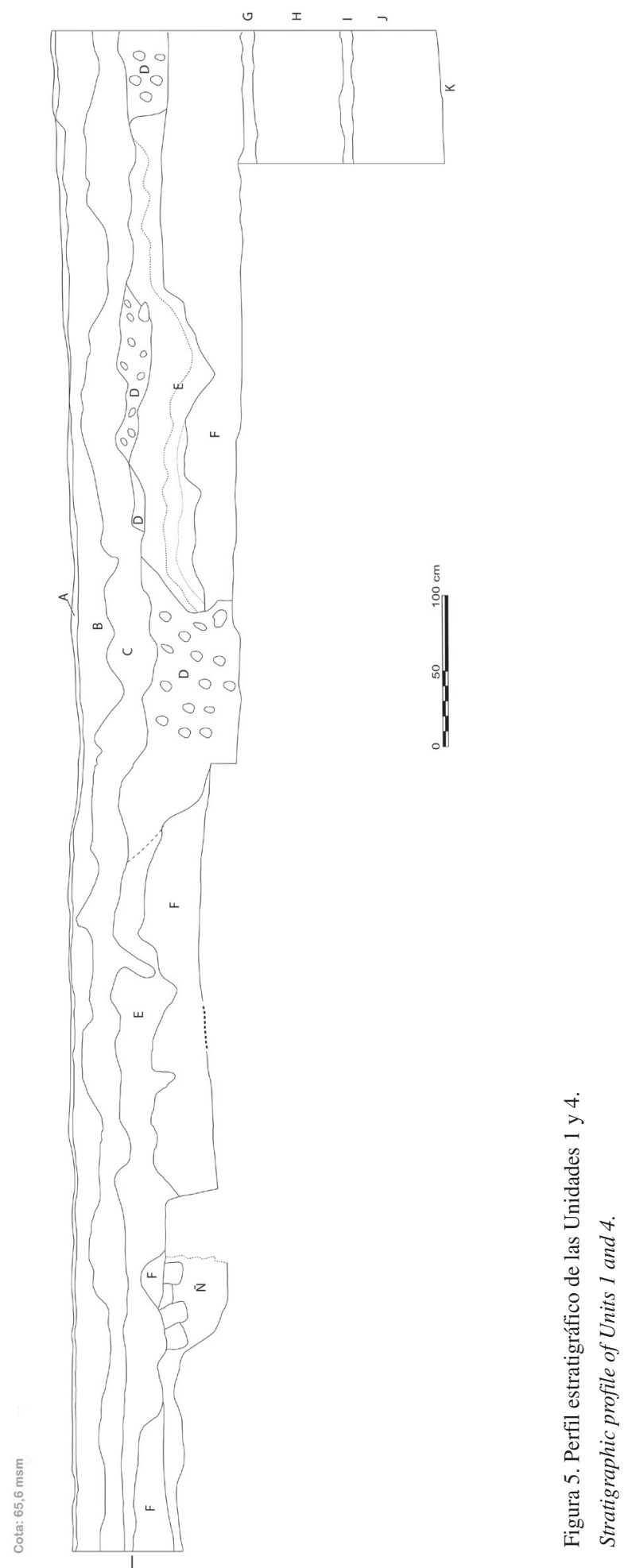




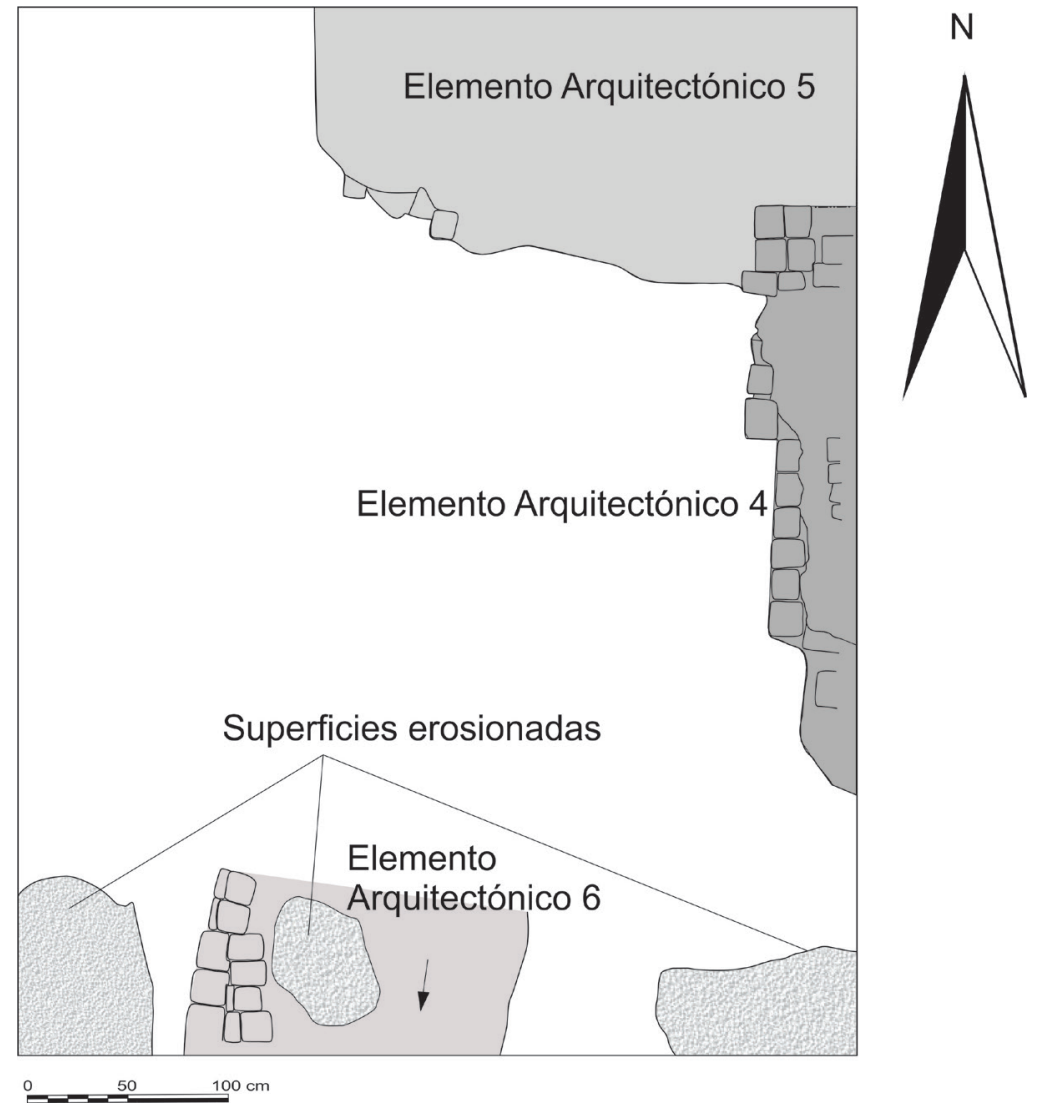

Figura 6. Plano del Piso de Ocupación - Capa G en la Unidad de Excavación 1. Plan of Layer G - Occupational Floor in Unit 1.

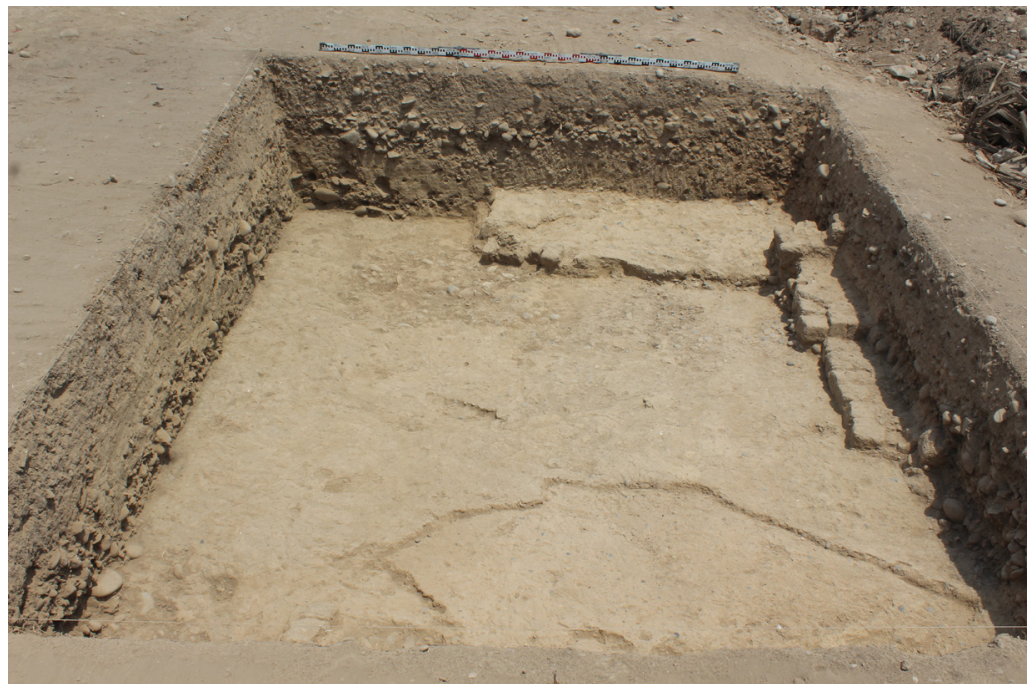

Figura 7. Vista del Piso de Ocupación - Capa G en la Unidad de Excavación 1. View of G Layer - Occupational floor in Excavation Unit 1. 


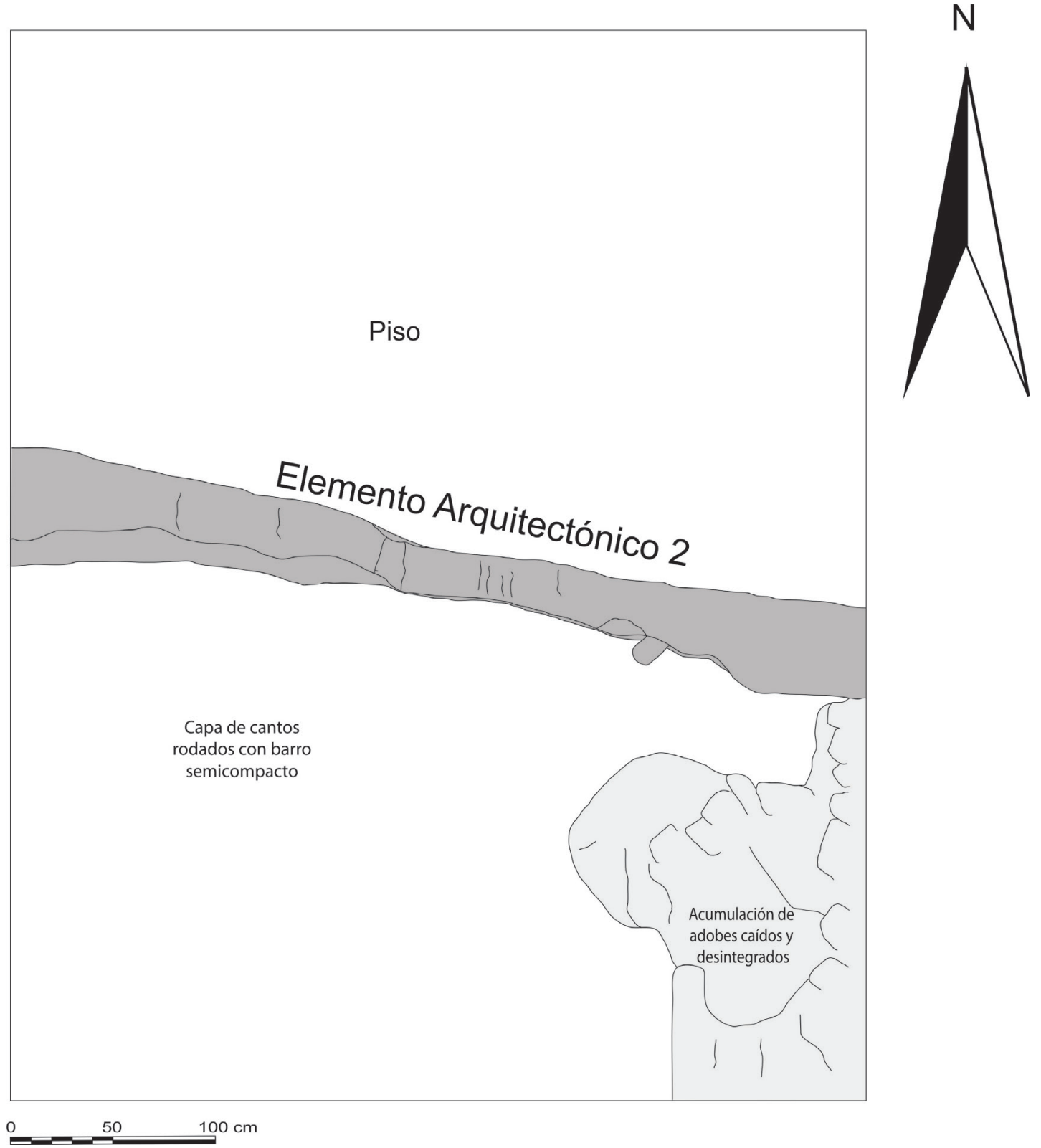

Figura 8. Plano del Piso de Ocupación - Capa G en la Unidad de Excavación 4.

Plan of G Layer - Occupational Floor in Excavation Unit 4.

banquetas nos sugiere que nos encontramos dentro de lo que en Huaca Pucllana se ha definido como un "patio con estructuras escalonadas". Se trataría, por lo tanto, de un típico espacio formal Lima.

Con el objetivo de obtener las muestras para el análisis micromorfológico, se condujo una excavación limitada de 1 x $1 \mathrm{~m}$ en la esquina noroeste de la Unidad 1 (Figura 9). Esta excavación permitió registrar que el piso Capa $\mathrm{G}$ era de una composición limo-arcillosa compacta, de unos $7 \mathrm{~cm}$ de espesor. Bajo esta capa, se encontraba un relleno de limo, arena y cantos rodados $($ Capa $\mathrm{H})$, de 50 a $58 \mathrm{~cm}$ de espesor, que cubría otro piso (Capa I), de composición similar a la Capa G y de un espesor de $8 \mathrm{~cm}$. Bajo este piso se halló otro relleno arquitectónico (Caja J), también compuesto por limo, arena y cantos rodados, de 45 a $54 \mathrm{~cm}$ de espesor. Éste cubría un tercer piso (Capa K) que no fue excavado. 


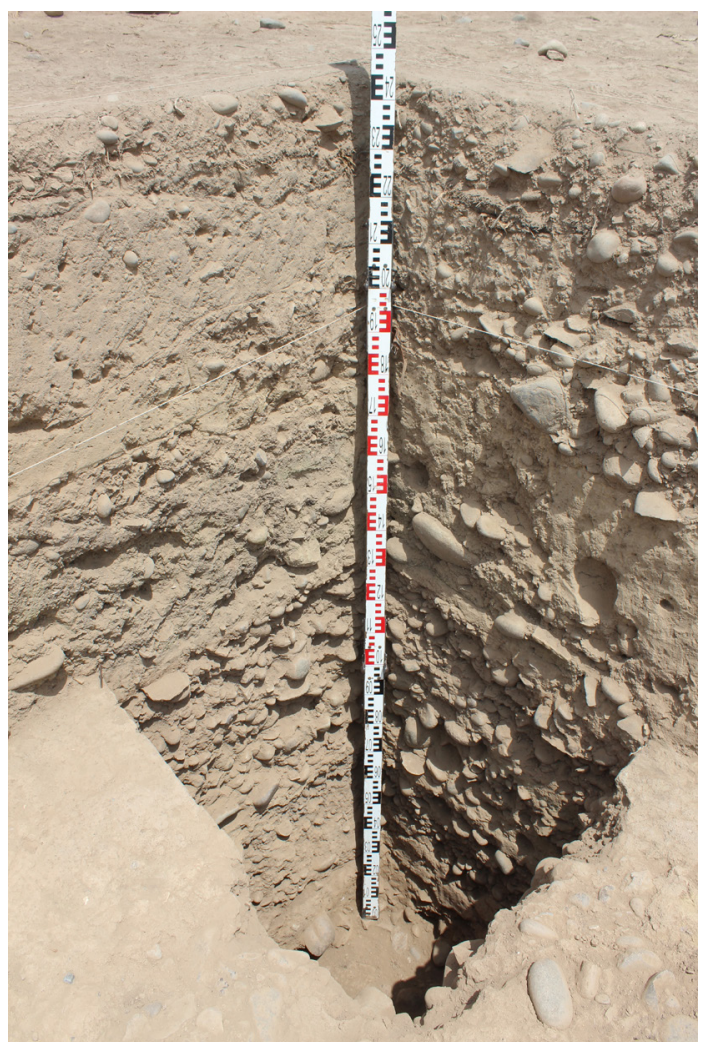

Figura 9. Vista del cateo de 1 x 1 m en la Unidad de Excavación 1.

View of $1 \times 1 \mathrm{~m}$ test pit at Unit 1 .

\section{El Análisis y los Resultados}

Con el objetivo de expandir nuestras posibilidades de reconstrucción de actividades en los espacios excavados, se decidió llevar a cabo un análisis micromorfológico de los pisos registrados en la Unidad 1. Para tal fin, se extrajeron cuatro muestras de los perfiles estratigráficos expuestos en el cateo llevado a cabo en la esquina noroeste de dicha unidad. Dos muestras (180 y 181) provienen del piso superior (Capa G) y dos $(182,183)$, del piso medio (Capa I) (Figura 10). De acuerdo con los requerimientos de laboratorio, las muestras fueron retiradas como bloques en los que se conservó la estructura sedimentaria de pisos y capas. Los bloques extraídos pesaron de 400 a 850 gr (Figura 11).

En el laboratorio, las muestras fueron secadas en un horno para retirar todo rastro de humedad y, posteriormente, impregnadas con una resina adherente en vacío. Luego de este tratamiento, fueron "rebanadas" hasta obtener láminas de 30 micrómetros de grosor que fueron colocadas entre placas de vidrio de 11,5 x 7,5 cm (Figura 12).

Estas secciones delgadas fueron analizadas con un microscopio polarizador Leica DMLP con un rango de magnificación de x10 a x 400, con luz polarizadora plana (PPL), luz polarizadora cruzada (XPL) y, en ciertos casos, con luz incidental oblicua (OIL). Cada fuente de luz permitió la identificación microscópica de diferentes elementos entre componentes minerales y orgánicos. El registro microfotográfico se realizó con una cámara Leica $\mathrm{DFC}_{295}$ anexa al microscopio, usando el programa de procesamiento fotográfico Leica.

El análisis de las cuatro muestras ofreció resultados relacionados con las características de la composición de la masa sedimentaria y su microestructura, sus rasgos edáficos y la existencia de minerales gruesos y componentes orgánicos (Tabla 1).

Un primer hallazgo fue la existencia de diferencias significativas en los procesos de deposición de sedimentos tanto entre las muestras como al interior de las mismas. Esto llevó a subdividir la microestratigrafía de los pisos registrados en diferentes microfacies. Así, en el caso del piso superior (Capa G), la muestra 180 fue dividida en tres microfacies (de arriba abajo, 180/1, 180/2 y 180/3), mientras que la muestra 181 fue dividida en dos (181/1 y 181/2). En el caso del piso medio (Capa I), la muestra 182 se dividió en dos microfacies (182/1 y 182/2), al igual que la muestra 183 (183/1 y 183/2) (Figura 11).

Un ejemplo de las diferencias detectadas fue que, en el caso de las muestras 180/2, 182/2 y 183/2, la proporción de materiales gruesos vs sedimentos finos era de 3:7, mientras que en las muestras 180/1, 182/1 y $183 / 1$, se incrementaba la presencia de materiales gruesos hasta estar en proporción de 1:1 o 2:3.

La microestructura de las muestras es bastante similar, con rasgos edáficos moderados a consolidados. Solo en el caso de la muestra 183/2 se ve un desarrollo débil. Es común, por otro lado, la disposición intercalada de "agregados en bloque" y "poros en canal". Todas las muestras presentan el desarrollo de nódulos redoximórficos en la matriz. Se trata de un rasgo edafológico que se forma en los bordes exteriores de partículas subangulares debido a la reducción u oxidación de sesquióxidos de hierro (Figura 13).

En la muestra 182/1 se encontraron además revestimientos particulados dispuestos en forma laminada en los poros en canal, mientras que en las muestras 180/2 y 181/2 se hallaron rellenos de los 


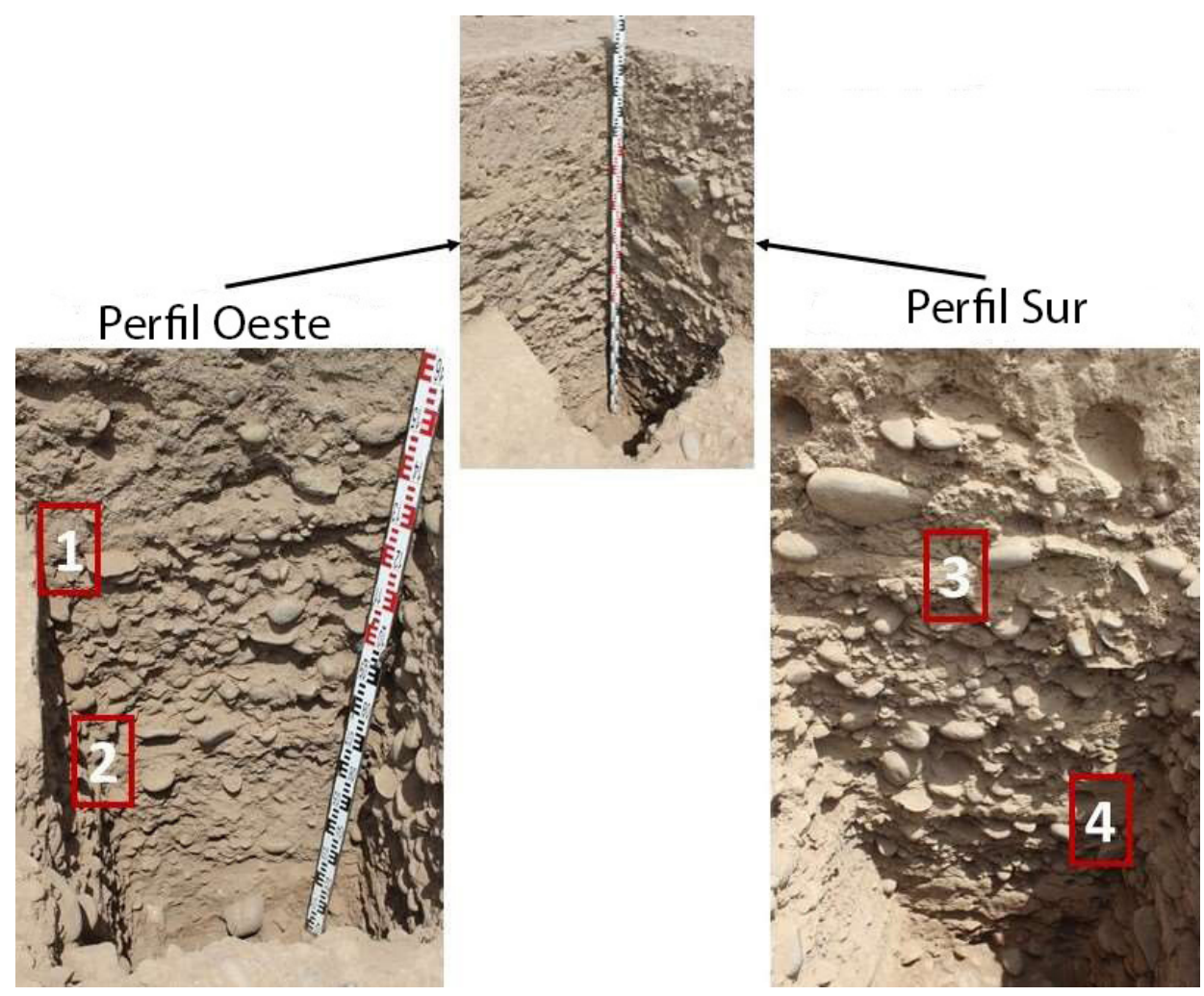

Figura 10. Ubicación de los puntos de extracción de muestras para análisis micromorfológico.

View of the extraction loci of samples for micromorphological analysis.
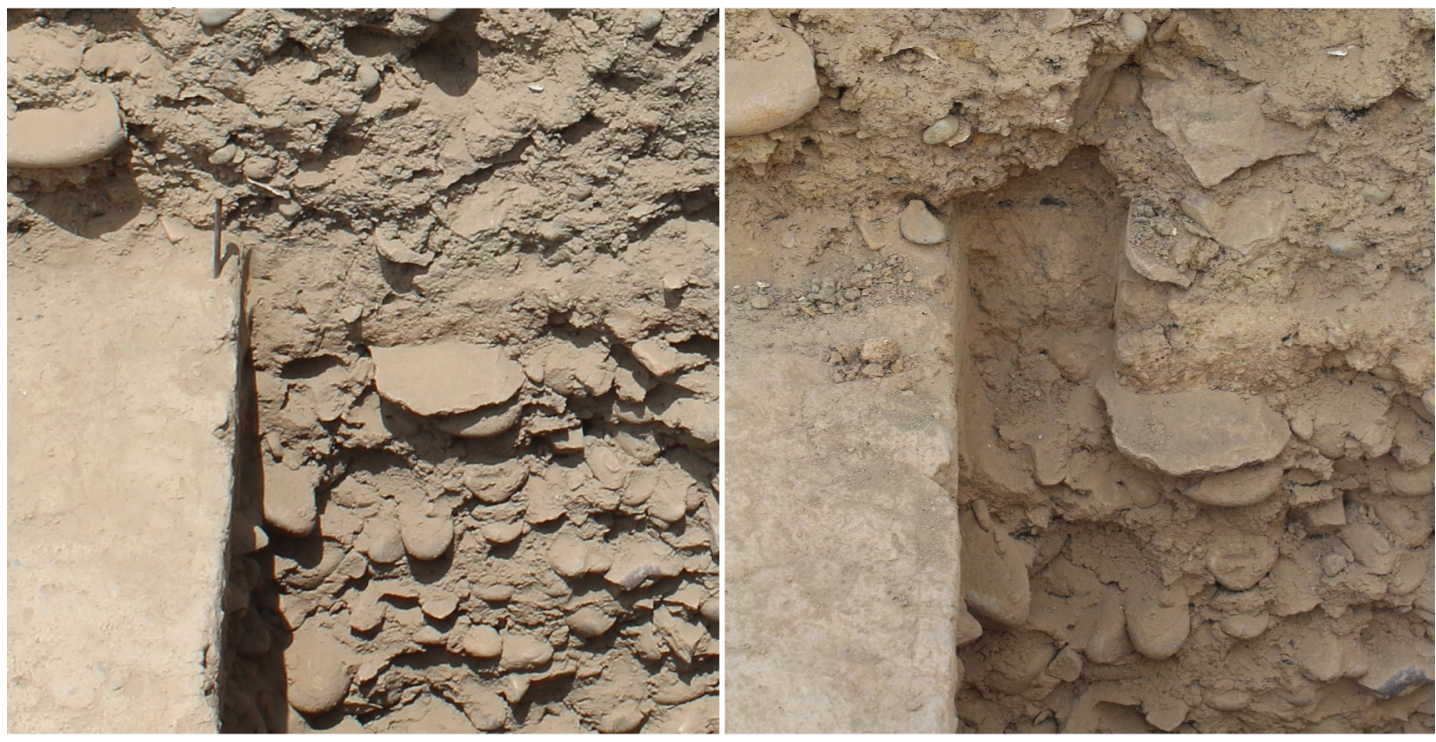

Figura 11. Vista de la ubicación de la muestra 180 antes y después de la extracción.

Location of Sample 180 before and after extraction. 


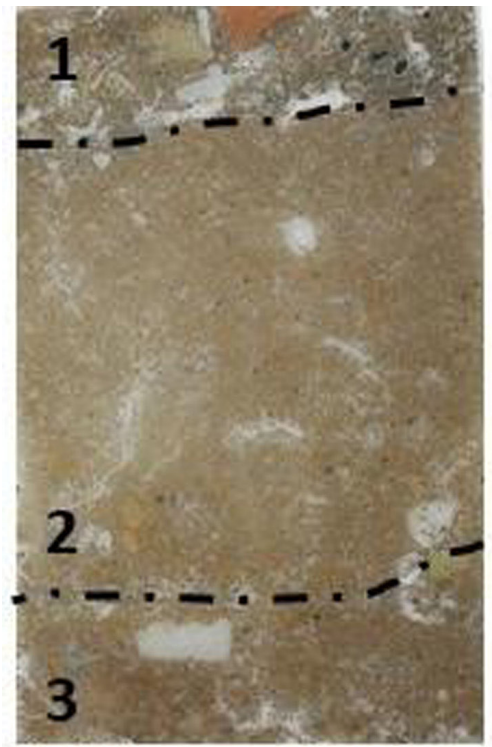

\section{Muestra 180}

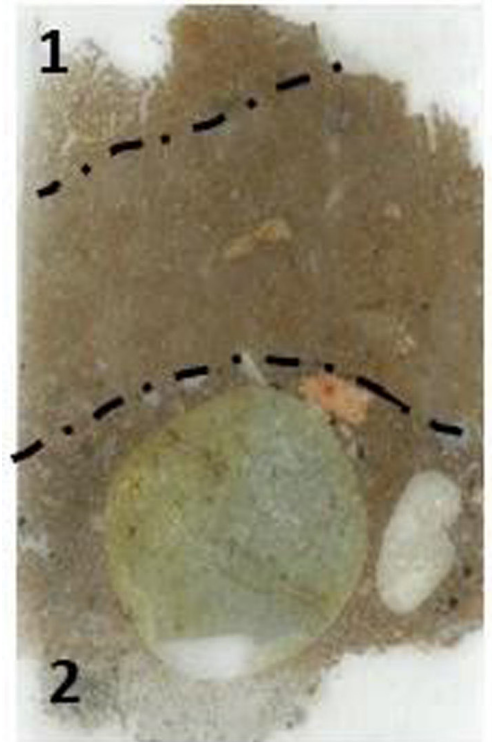

\section{Muestra 183}

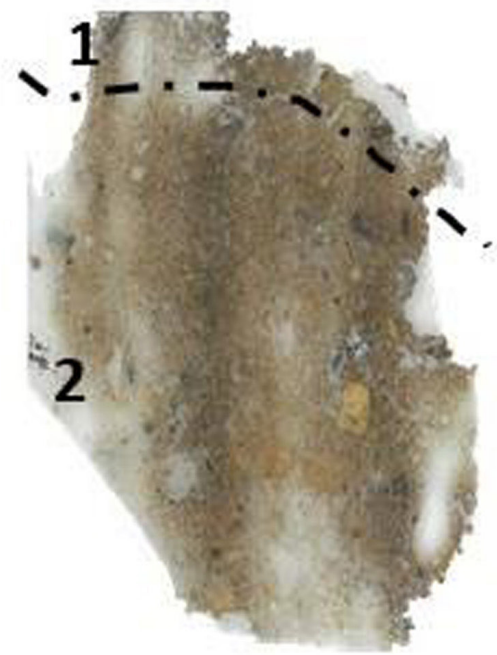

Muestra 181

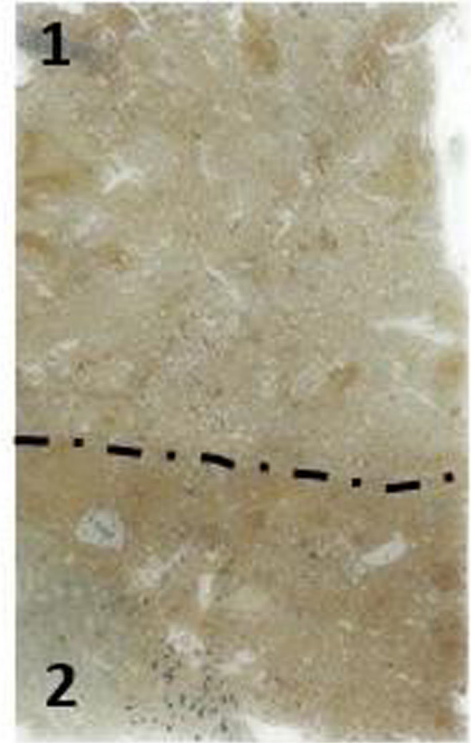

Muestra 182

Figura 12. Vista de las secciones delgadas provenientes de las muestras extraídas, con indicación de las unidades geomorfológicas.

View of thin sections from the soil samples, indicating their geomorphological units.

mismos sedimentos laminados dentro de la masa basal. Estos rellenos incluían microrrestos (de menos de 20 micrómetros) de carbón, nódulos redoximórficos y materia orgánica amorfa. Por su parte, las muestras 181/1 y 180/2 presentaban una apariencia "moteada" debido a estriaciones de granos y poros por presiones al interior de la matriz de coloides de arcilla.
El registro mineralógico señala la presencia de minerales del manto volcanoclástico local, con fragmentos subangulares y subredondeados de cuarzo, basalto, biotita y microclina. De manera excepcional, se registró una partícula de calcita de 2500 micrómetros en la muestra 183/2 junto con un fragmento parcialmente desintegrado de gabro (Figura 14). 


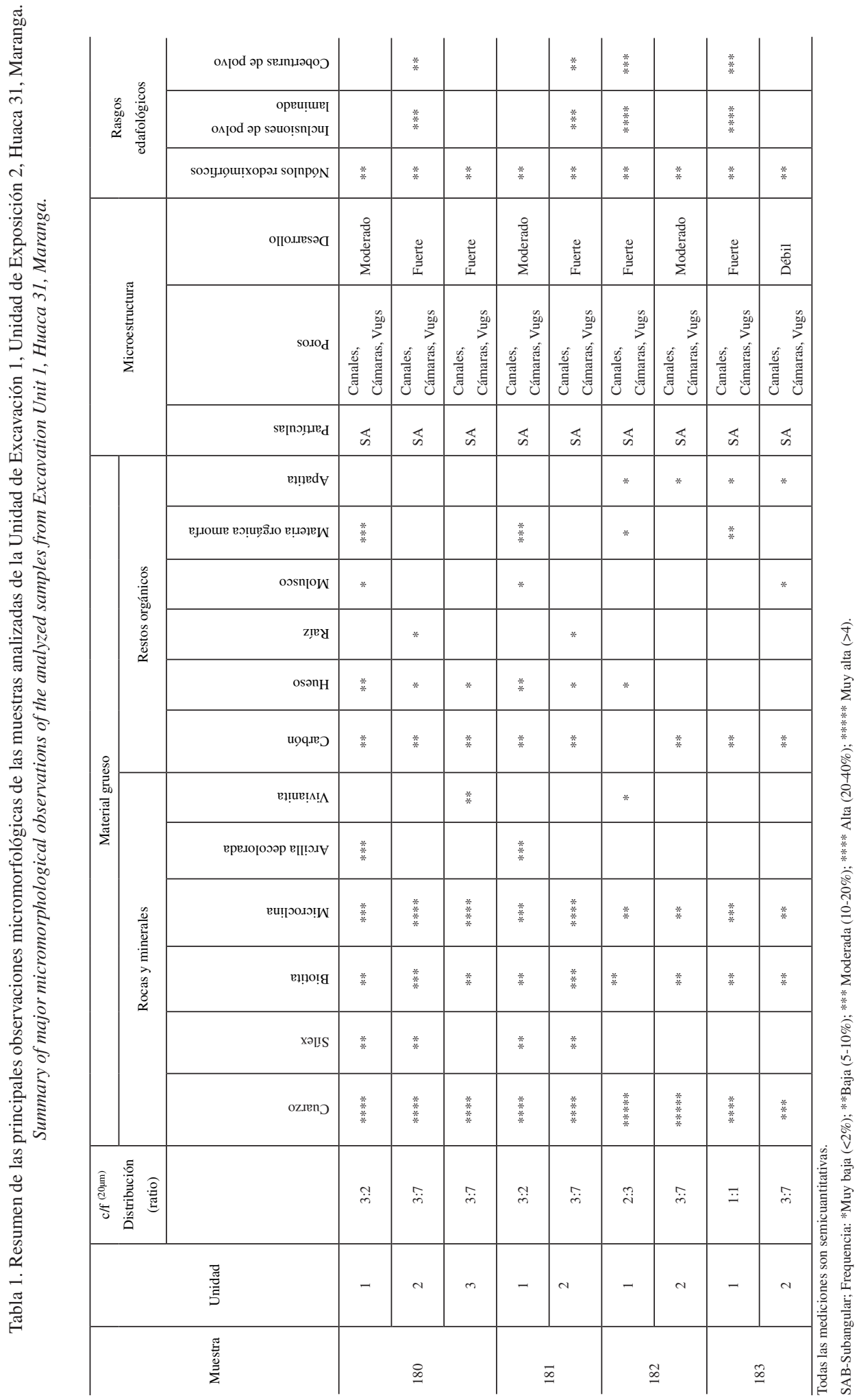


En relación con los restos de origen orgánico, fue posible la identificación de fragmentos deteriorados de moluscos en las muestras 180/1 y181/2, con la notable presencia de un pedazo de mayor tamaño en la muestra 180/2 (Figura 15). De igual forma, en todas las muestras se hallaron fragmentos de huesos en diferentes grados de deterioro, incluyendo algunos quemados (Figura 16). Las muestras del piso superior (180 y 181) presentan un menor grado de deterioro, algo que coincide con una mayor frecuencia de huesos quemados. Por otro lado, la mayor parte de las muestras, con excepción de la 182/2, presentó fragmentos de carbón. Por último, la muestra 183/1 presentó una alta frecuencia de materia orgánica amorfa.

Fue significativo también el hallazgo de una alta frecuencia de fitolitos fragmentados en la muestra 182/1 (Figuras 17 y 18). Estos fragmentos se encontraban dispuestos en forma paralela al borde, entre la masa basal y los rellenos laminados de los poros en canal.
La morfología de los fragmentos sugiere que se derivarían de algún tipo de pasto.

\section{Implicancias de los Resultados}

Los resultados obtenidos han permitido detectar actividades secuenciales sobre los pisos, que en varios casos incluyen la presencia de microartefactos.

En primer lugar, el registro en los pisos de inclusiones laminadas de sedimentos sugiere la mezcla de componentes conforme se añadía nuevo material que, al compactarse, iba formando una estratigrafía intercalada de agregados en bloque y poros en canal. A esto hay que añadir el registro de rellenos de partículas de arcilla que forman microcoberturas. Estas sugieren acarreos de partículas suspendidas (y luego decantadas) en agua, conforme se va dando también la alteración y lavado de las superficies originales. Esta actividad se estaría produciendo a partir de sucesivos eventos

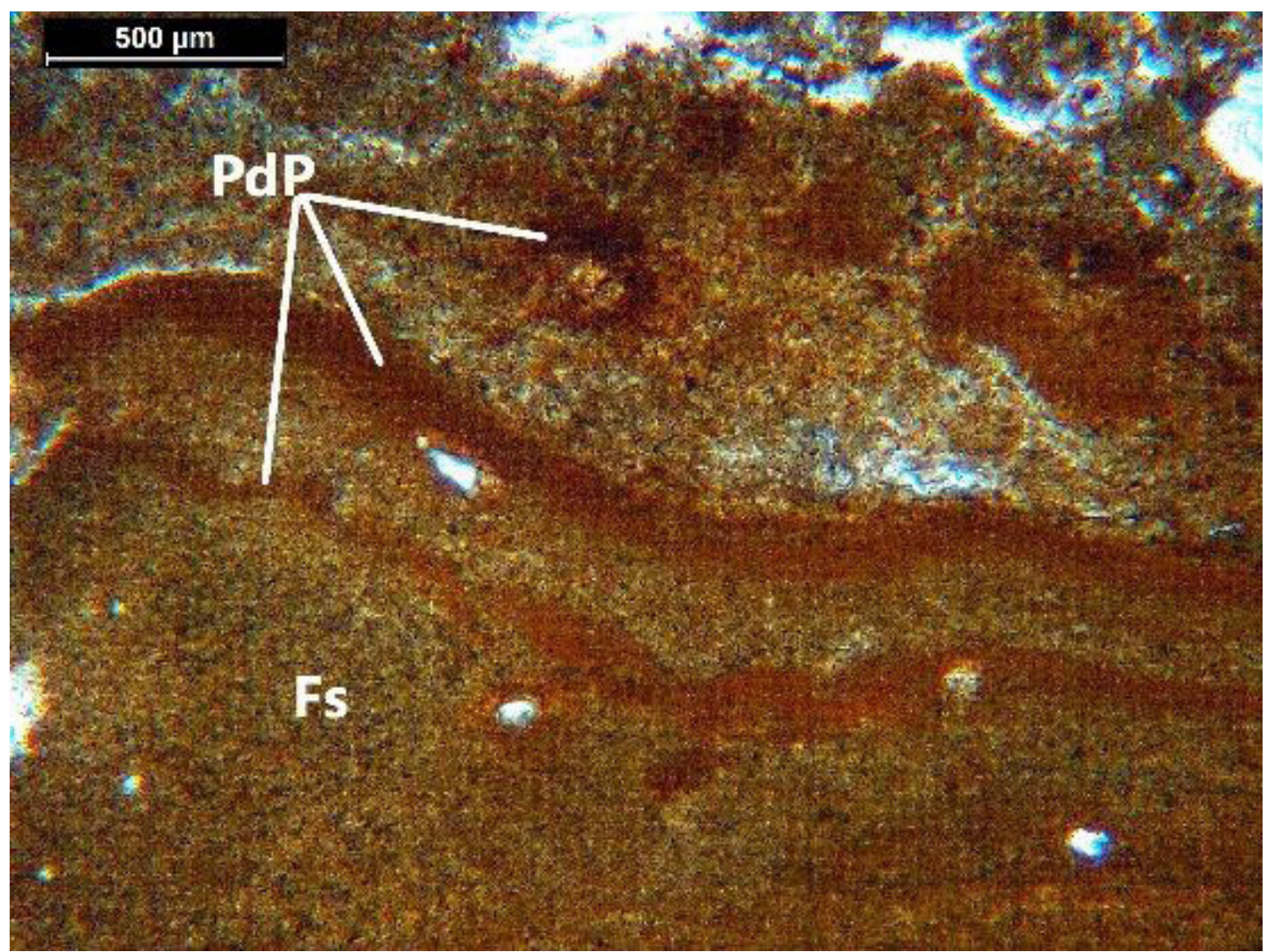

Figura 13. Detalle de desplazamiento postdeposicional de elementos sesquióxidos entre sedimentación fina en la muestra 183/1 (visto con luz polarizadora plana).

Detail of post-depositional displacement of sesquioxide features in fine sedimentation (Sample 183/1) (seen with PPL). 


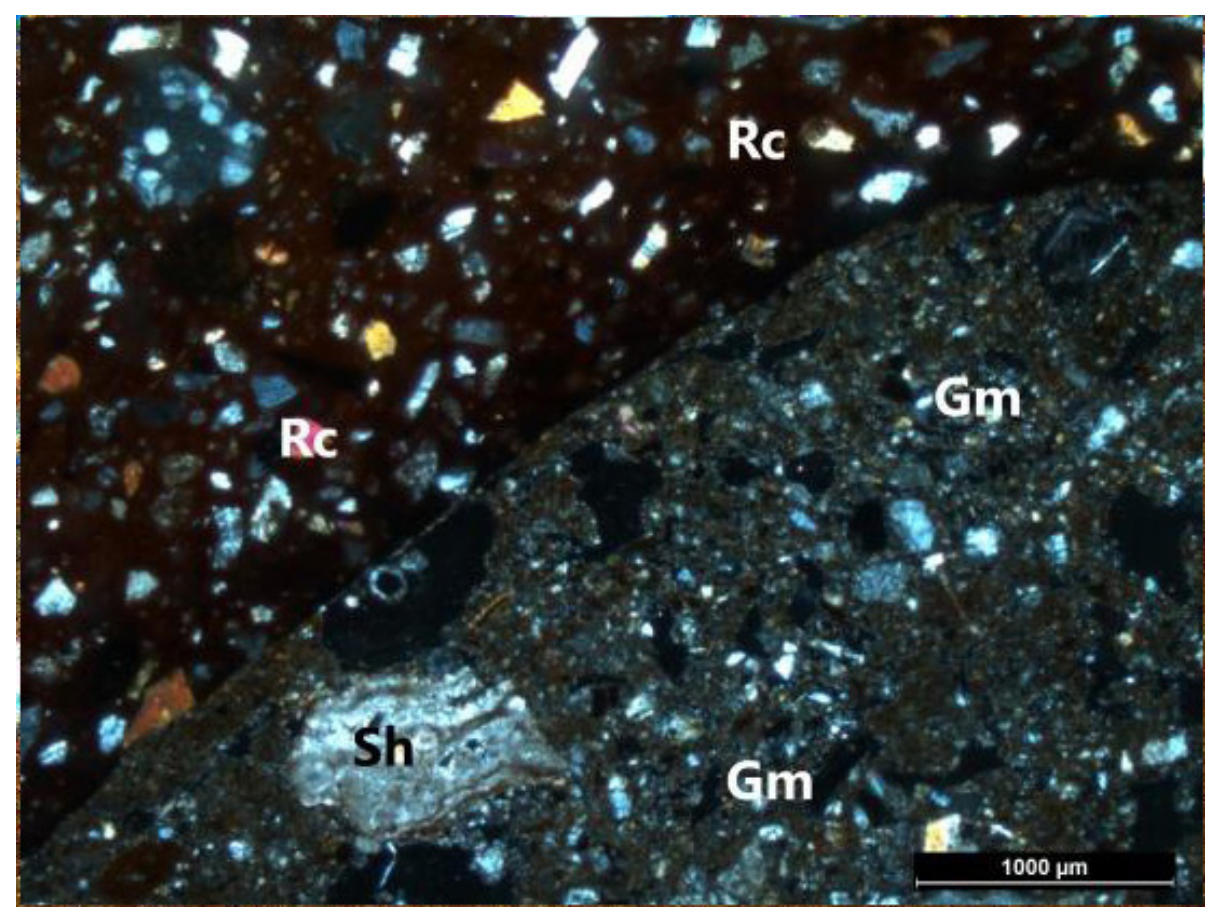

Figura 14. Arcilla rubificada (Rc) dentro de la masa basal $(\mathrm{Gm})$ que contiene fragmentos de moluscos (Sh) (visto con luz polarizadora cruzada).

Rubified clay $(R c)$ within the groundmass $(\mathrm{Gm})$ that contains several small shell (Sh) fragments (Seen with XPL).

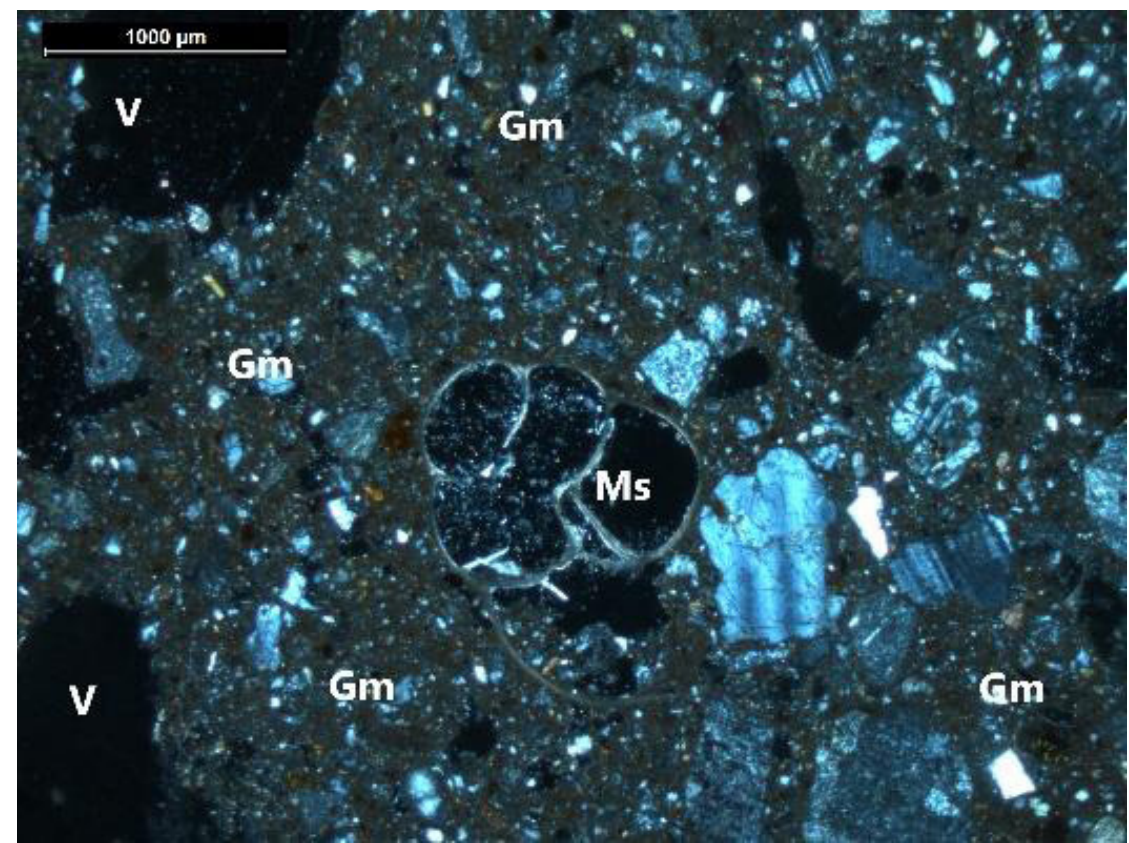

Figura 15. Detalle de un fragmento de valva de molusco entre masa basal $(\mathrm{Gm})$, cerca a poros en canal (V) en la muestra 180/2 (visto con luz polarizadora cruzada).

Detail of a shell fragment in the groundmass $(\mathrm{Gm})$, with visible chamber voids $(\mathrm{V})$ in Sample 180/2 (Seen with XPL). 


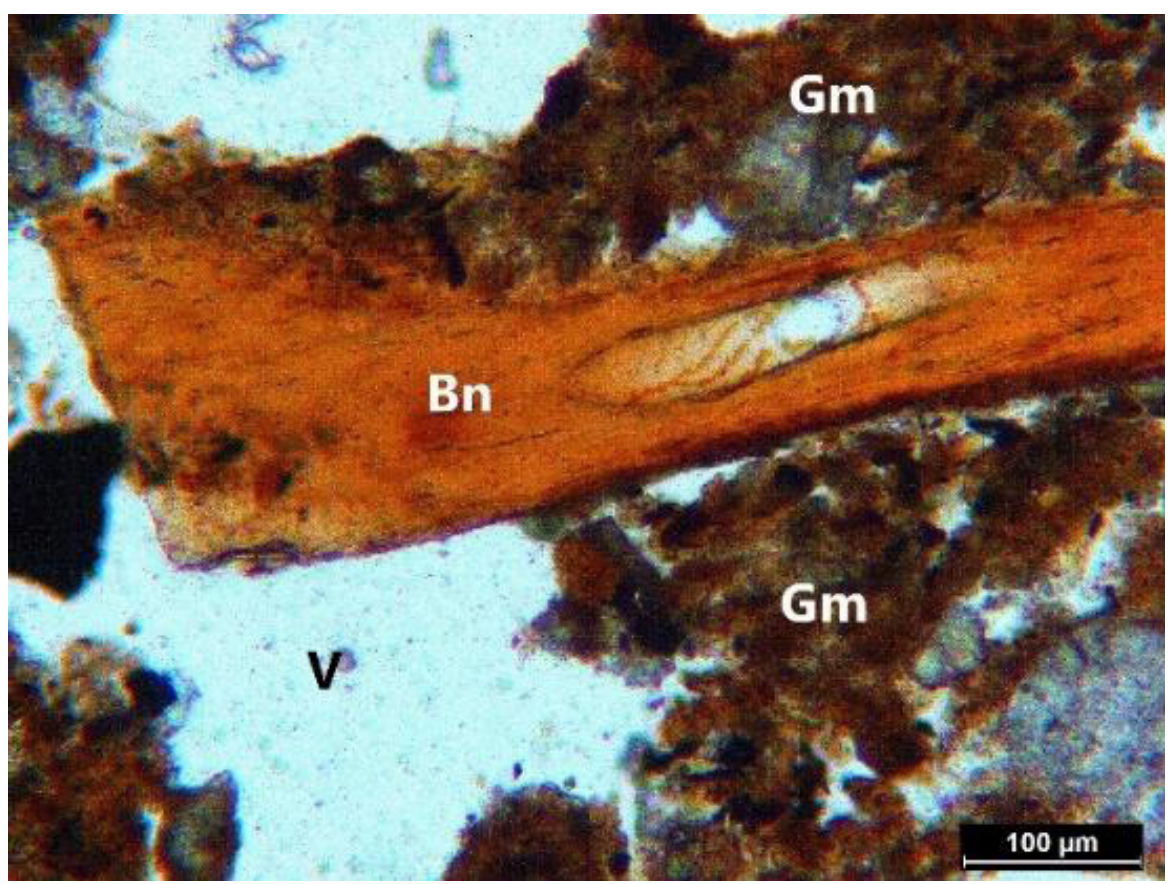

Figura 16. Detalle de hueso quemado entre masa basal (GM) y poros en canal (V). Muestra 182/2 (visto con luz polarizadora plana).

Detail of burnt bone between ground mass (GM) and voids (V). Sample 182/2 (Seen with PPL).

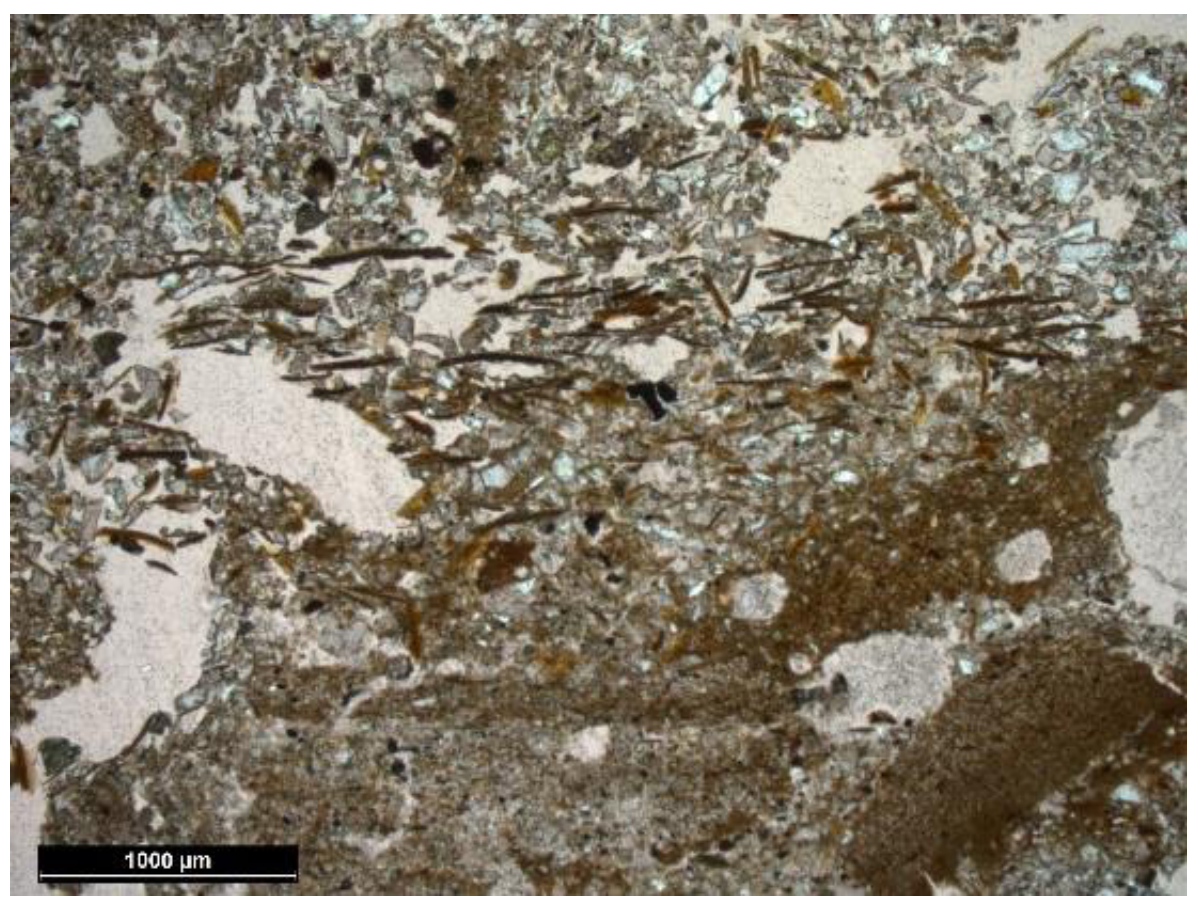

Figura 17. Laminación de material fino en la masa basal de la muestra 182/1 con fitolitos fragmentados en alineamientos paralelos (visto con luz polarizadora plana).

Laminations of fine material in the ground mass of sample 182/1 with fragmented phytoliths in parallel alignment (Seen with PPL). 


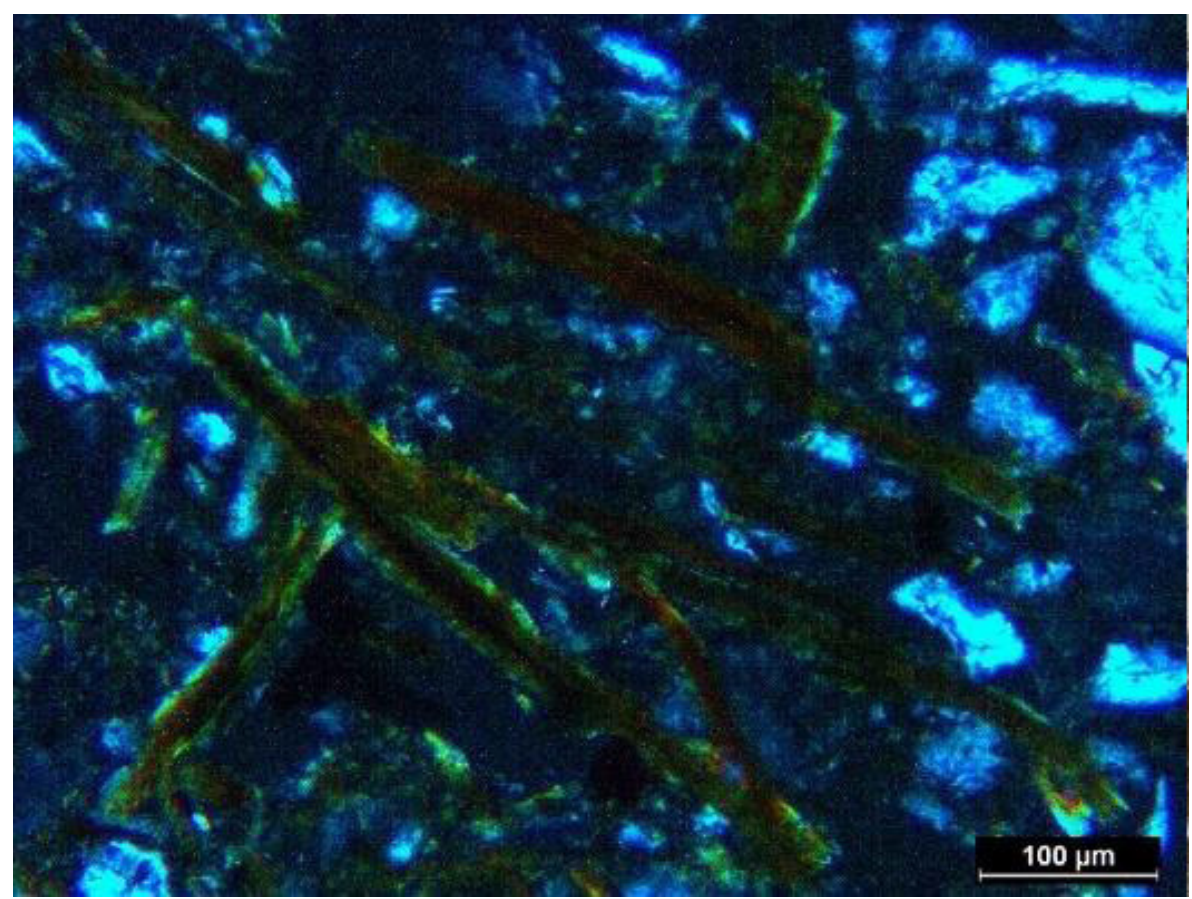

Figura 18. Detalle de fitolitos alineados. Muestra 182/1 (visto con luz polarizadora cruzada).

Increased magnification of the parallel aligned phytoliths in sample 182/1 (Seen with XPL).

de precipitación y formación de aniegos (Durand et al. 2010). La explicación de estos eventos estaría en actividades de lavado de superficies como parte del mantenimiento de los pisos y la reducción de polvo. Se trata de procesos edafológicos de naturaleza antrópica, cuyas implicancias en el entendimiento de los contextos de ocupación serán materia de futuras indagaciones.

En estos procesos de limpieza, por otro lado, quedaron atrapadas en los sedimentos micropartículas de artefactos y componentes orgánicos. Así, se registró una alta frecuencia de huesos fragmentados en varios estados de degradación, con evidencias de quema en gran proporción, junto con evidencia de restos malacológicos y materia orgánica amorfa. Esto, junto con la presencia de carbón y arcilla decolorada, nos indica actividades de limpieza luego de eventos de quema (Banerjea et al. 2015) que incluyeron bienes alimenticios. El alcance de las muestras no permite establecer de manera más precisa el tipo de actividades, pero podría tratarse de contextos comparables como aquellos registrados en Pucllana o Cajamarquilla, que incluyen el procesamiento y consumo de alimentos. En este caso, sin embargo, no se trataría de actividades relacionadas directamente con un evento constructivo.

En el caso del piso medio, fue notable además el registro de fitolitos no carbonizados que sugieren una cubierta vegetal del piso. Puede contemplarse la posibilidad de que se trate de un alfombrado de esteras. De ser así, se nos abre la posibilidad de incorporar, en el análisis espacial de este tipo de arquitectura, la presencia de instalaciones muebles y su relevancia en la organización de las actividades.

Así, puede concluirse que el análisis micromorfológico indica que los pisos tuvieron múltiples fases de desarrollo. Dentro de estas fases, los sedimentos laminados revelan la formación de diferentes superficies sobre las que se aplastaron micropartículas que nos revelan la naturaleza de las actividades allí llevadas a cabo, destacando la posibilidad de una cobertura vegetal en el piso medio y actividades de cocción de alimentos (y su posterior limpieza) en el piso superior.

\section{Conclusiones y Perspectivas}

El estudio aquí presentado tiene claramente un carácter exploratorio. Como tal, permite comprobar el potencial informativo de los análisis micromorfológicos para contextos deposicionales de arquitectura formal como pisos, banquetas o plataformas que por lo general se presentan "limpios" de restos a nivel macroscópico. Tomando en consideración las conocidas condiciones de preservación de restos del desierto andino, es de 
esperar que el desarrollo de la microestratigrafía y la micromorfología en este territorio ofrezca un mayor abanico de posibilidades de registro.

Este estudio plantea, por otro lado, nuevos requerimientos para poder convertirse en una herramienta útil en la reconstrucción de áreas de actividad. En primer lugar, se hace necesario trabajar con espacios delimitados, tanto por componentes arquitectónicos (muros, banquetas, etc.) como por elementos e instalaciones identificados macroscópicamente. En otras palabras, se requiere de la mayor información contextual que contribuya al entendimiento de la información microestratigráfica.

En segundo lugar, la reconstrucción de áreas de actividad requiere de una adecuada estrategia de muestreo. Cada muestra representa un pedazo mínimo de espacio, y puede resultar peligroso generalizar tipos de actividades a partir de un número reducido de ellos. En ese sentido, la primera condición (de contar con un contexto o espacio definido) es fundamental, porque nos permite orientar dónde tomar las muestras y cuántas de ellas pueden ser representativas de acuerdo con el tamaño del área a estudiar.

En tercer lugar, la micromorfología tiene el potencial de registrar secuencias de cambios de tiempos muy cortos. En tal sentido, resulta importante incluir, tanto en el muestreo como en la concepción del análisis, una perspectiva diacrónica, que aborde los posibles cambios en el uso de los espacios a través de tiempos cortos o largos.

En cuarto lugar, el crecimiento de la microestratigrafía y la micromorfología en los Andes dependerá también de la concurrencia de diferentes competencias, que permitan la correcta identificación de minerales, fitolitos, granos de polen, componentes orgánicos y todo tipo de microrrestos posiblemente involucrados. Se trata además de técnicas en las que la identificación se basa fundamentalmente en registros ópticos. En ese sentido, la inclusión de análisis complementarios de identificación química o mineralógica de las mismas muestras o contextos permitirá también ampliar nuestras perspectivas de interpretación.
En el caso concreto de la Cultura Lima, los avances en el conocimiento de los conjuntos arquitectónicos que circundan los edificios públicos han permitido reconocer diferentes tipos de espacios como patios hipóstilos con banquetas, recintos alienados, además de sistemas de accesos con pasadizos y rampas. Existe, por lo tanto, una diversidad de espacios que suelen presentarse como conjuntos delimitados con sistemas de accesos singulares. Se trata, por lo tanto, de un escenario promisorio para medir la posibilidad de identificación de diferentes áreas de actividad y sus cambios y continuidades a lo largo del tiempo.

La microestratigrafía y la micromorfología han probado su relevancia en el avance del registro arqueológico en otras partes del mundo. El área andina es, sin duda, un territorio propicio para su expansión y la costa central, durante la época Lima, ofrece los contextos arquitectónicos propicios para dicha expansión.

Agradecimientos: El presente estudio pudo llevarse a cabo gracias a la obtención de una de las becas ofrecidas por la Dirección Académica de Investigación de la Pontificia Universidad Católica del Perú para el año 2017. En el campo, contamos con el apoyo de la División de Arqueología del Patronato Parque de las Leyendas (PATPAL) en cuyos terrenos se encontraba nuestra área de estudio. Mención especial a su directora, Lucénida Carrión, y a su jefa de gabinete, Gisela Tuesta. Giusseppe Alva, Ruth Garay, Ariadna Gómez, Rodrigo Luna, Manuel Morón, Faustina Orihuela, Marcia Orrego y Kely Ortega, estudiantes de la Universidad Mayor de San Marcos y de la Universidad Católica participaron de las excavaciones en la Unidad 1. Los hermanos Viviana e Igor Siveroni colaboraron generosamente en el traslado de las muestras de Lima a los laboratorios de Reading en el Reino Unido. A todas las personas e instituciones mencionadas, nuestro sincero agradecimiento. Finalmente, el manuscrito original fue significativamente mejorado gracias a las observaciones y sugerencias de los dos evaluadores anónimos asignados por la Revista.

\section{Referencias Citadas}

Amadio, M. 2019. Tracing post-depositional processes and preservation of architectural material deposits in the semiarid environment of southern Cyprus: A micromorphological approach. Journal of Archaeological Science: Reports 27:101986.

Balbo, A., M. Madella, A. Vila y J. Estévez 2010. Micromorphological perspectives on the stratigraphical excavation of shell middens: a first approximation from the ethnohistorical site Tunel VII, Tierra del Fuego (Argentina). Journal of Archaeological Science 37 (6):1252-1259.
Banerjea R., M. Bell, W. Matthews y A. Brown 2015. Applications of micromorphology to understanding activity areas and site formation processes in experimental hut floors. Archaeological and Anthropological Sciences 7:89-112.

Boivin, N. 2000. Life rhythms and floor sequences: excavating time in rural Rajasthan and Neolithic Catalhöyuk. World Archaeology 31 (3):367-388.

Carrión, L. 2006. Informe final del Proyecto de Evaluación Arqueológica en el Área Noreste del Parque de las Leyendas. Informe inédito presentado al Instituto Nacional de Cultura, Lima, Perú. 
Courty, M. 1992. Soil micromorphology in archaeology. Proceedings of the British Academy 77:39-59.

Durand N., C. Monger y M. Canti 2010. Calcium Carbonate Features. En Interpretation of Micromorphological Features of Soils and Regolith, editado por G. Stoops, V. Marcelino y F. Mees, pp. 149-194. Elsevier, Londres.

Engel, F. 1987. De las Begonias al Maíz: Vida y Producción en el Perú Antiguo. Universidad Nacional Agraria La Molina, Lima.

Flores, I. 2005. Pucllana, Esplendor de la Cultura Lima. Instituto Nacional de Cultura, Lima.

Flores, I., P. Vargas, J. Ccencho y H. Silvera 2012. Los patios con estructuras escalonadas de Huaca Pucllana: Caracterización y función de una arquitectura ceremonial Lima. Arqueología y Sociedad 25:35-36.

Franco, R. y P. Paredes 2001. El templo viejo de Pachacamac. Nuevos aportes al estudio del Horizonte Medio. Boletín de Arqueología PUCP 4:607-630.

Friesem, D., E. Friesem, G. Tsartsidou, P. Karkanas y R. ShahackGross 2013. Where are the roofs? A geo-ethnoarchaeological study of mud brick structures and their collapse processes, focusing on the identification of roofs. Archaeological and Anthropological Sciences 6:73-92.

Goldberg, P. 1983. Applications of micromorphology in archaeology. En Soil Micromorphology, editado por P. Bullock y C. Murphy, pp. 139-150. Academic Publishers, Berkhamsted.

Goodman-Elgar, M. 2008. Evaluating soil resilience in long-term cultivation: a study of pre-Columbian terraces from the Paca Valley, Peru. Journal of Archaeological Science 35:3072-3086.

Hutchinson, T. 1873. Two years in Peru, with Exploration of its Antiquities. Tomo I. Gilvert and Rivington, Londres.

Jijón y Caamaño, J. 1949. Maranga. Contribución al Conocimiento de los Aborígenes del Valle del Rímac, Perú. La Prensa Católica, Quito.

Karkanas, P. y P. Goldberg 2007. Micromorphology of sediments: Deciphering archaeological context. Israel Journal of Earth Sciences 56:63-71.

Kroeber, A. 1956. Proto Lima. A Middle Period Culture of Peru. Fieldiana Anthropology 54 (1). Chicago Natural History Museum, Chicago.

La Motta, V. y B. Schiffer 1999. Formation processes of house floor assemblages. En The Archaeology of Household Activities, editado por P. Allison, pp. 19-29. Routledge, Londres.

Lumbreras, L. 2011. Maranga. Estudio sobre Lima Prehispánica según Jacinto Jijón y Caamaño. PETROPERÚ-Municipalidad Metropolitana de Lima, Lima.

Macphail, R., M. Courty y P. Goldberg 1989. Soil micromorphology in archaeology. Endeavour 14 (4):163-171.

Maquera, E. y M. Esteban 2014. Investigaciones arqueológicas en Catalina Huanca, un asentamiento de la sociedad Lima del Horizonte Medio. Boletín de Arqueología PUCP 18:81-104.

Matthews, W. 2005. Micromorphological and microstratigraphic traces of uses and concepts of space. En Inhabiting Çatal Höyük. Reports from the 1995-1999 Seasons, editado por I. Hodder, pp. 355-398. McDonald Institute of Archaeological Research - British Institute of Archaeology, Ankara.

Matthews, W., C.A.I. French, T. Lawrence, D.F. Cutler y M.K. Jones 1997. Microstratigraphic Traces of Site Formation Processes and Human Activities. World Archaeology 29 (2):281-308.
Méndez, C., A. Nuevo, O. Reyes, I. Ozán, C. Belmar y P. López 2018. The initial peopling of Central Western Patagonia (southernmost South America): Late Pleistocene through Holocene site context and archaeological assemblages from Cueva de la Vieja site. Quaternary International 473:261-277.

Middendorf, E. W. 1973 [1894]. Perú. Observaciones y Estudios del País y sus Habitantes durante una Permanencia de 25 años. Tomo II. La Costa. Universidad Nacional Mayor de San Marcos, Lima.

Narváez, J. y P. Manrique 2014. Arquitectura lima en Maranga. En Arqueología. Catorce años de Investigaciones en Maranga, editado por L. Carrión y J. Narváez, pp. 95-119. Municipalidad de Lima Metropolitana, Lima.

Paredes, J. 1992. Cerro Culebras: Nuevos aportes acerca de una ocupación de la Cultura Lima-Costa Central del Perú. Gaceta Arqueológica Andina 22:51-62.

Ríos, N. 2008. Restos de actividades rituales en la segunda etapa constructiva de una plaza Lima Tardío: un caso en Pucllana. Tesis de licenciatura para optar el título de arqueólogo, Facultad de Ciencias Sociales, Universidad Nacional Mayor de San Marcos, Lima.

Schiffer, M.B. 1995. Behavioral Archaeology. First Principles. The University of Utah Press, Salt Lake City.

Segura, R. 2001. Rito y Economía en Cajamarquilla: Investigaciones Arqueológicas en el Conjunto Arquitectónico Julio C. Tello. Pontificia Universidad Católica del Perú, Lima.

Shillito, L., W. Matthews, M. J. Almond e I.D. Bull 2011. The microstratigraphy of middens: Capturing daily routine in rubbish at Neolithic Çatal Höyük, Turkey. Antiquity 85:1024-1038.

Stumer, L. 1954. The Chillón Valley of Perú. Excavation and Reconnaissance 1952-1952. Part 2. Archaeology 7 (4):220-228.

Uhle, M. 1998 [1910]. Acerca de las culturas tempranas de Lima y sus alrededores. En Max Uhle y el Perú Antiguo, editado por P. Kaulicke, pp. 231-254. Pontificia Universidad Católica del Perú, Lima.

Vargas, P. 2012 Secuencia constructiva de la Gran Pirámide de Huaca Pucllana en sus tiempos finales. Investigaciones sociales 16 (28):303-311.

Vega Centeno, R., C. Toledo, L. Huamán y G. Alexandrino 2021. Examen de un perfil estratigráfico Lima en Maranga. Latin American Antiquity 32 (3):655-661.

Villagran, X. 2016. Micro Categories Analysis: the Use of Micromorphology for Site Formation and Taphonomic Studies in Archaeological Sites. En The Origins of Food Production, editado por N. Sanz, pp. 326-329. UNESCO, Ciudad de México.

Villagran, X. 2019. The Shell Midden Conundrum: Comparative Micromorphology of Shell-Matrix Sites from South América. Journal of Archaeological Method Theory 26:344-395.

Villagran, X., A. Balbo, M. Madella, A. Vila y J. Estevez 2011. Experimental micromorphology in Tierra del Fuego (Argentina): building a reference collection for the study of shell middens in cold climates. Journal of Archaeological Science 38:588-604.

Villagran, X. y P. Giannini 2014. Shell mounds as environmental proxies on the southern coast of Brazil. The Holocene 24 (8):1009-1016.

Willey, G.R. 1943. Excavations in the Chancay Valley. En Archaeological Studies in Perú, 1941-42, editado por W.D. Strong, G.R. Willey y J.M. Corbett, pp. 123-197. Columbia University Press, Nueva York. 